\title{
The flow around a surface mounted cube: a characterization by time-resolved PIV, 3D Shake-The-Box and LBM simulation
}

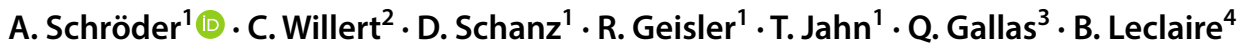

Received: 19 March 2020 / Revised: 22 June 2020 / Accepted: 13 July 2020 / Published online: 9 August 2020

(c) The Author(s) 2020

\begin{abstract}
The flow around a surface mounted cube with incoming turbulent or laminar boundary layer has been topic of many experimental and numerical investigations in the past decades. Despite its simple geometry the flow generates a set of complex vortical structures in front and around the cube, includes flow separation at the three front plane edges with corresponding subsequent shear layer dynamics enveloping recirculation zones. Downstream of the cube a large unsteady flow separation region is present which is associated with typical quasi-periodic bluff-body wake dynamics. Therefore the flow configuration is well suited to enhance the understanding of similar unsteady and separated flow phenomena in many aerodynamic and engineering applications. In the present experimental investigation we aim at resolving a large spectrum of spatial and temporal scales in the flow around a cube with incoming laminar and turbulent boundary layers by using the most recent developments of dense 3D Lagrangian particle tracking (LPT) and high resolution TR-PIV for Reynolds numbers based on cube size in the range $\operatorname{Re}_{H}=U_{\infty} H v^{-1}=2000-8000$. The results documented in the present paper consist of snapshots and the analysis of long time-series of highly resolved 3D and 2D velocity fields suited to enhance the understanding of coherent structure dynamics and of corresponding statistical Lagrangian and Eulerian flow properties. Premultiplied velocity spectra and 3D pressure distributions are calculated and discussed as well. Finally, the measurement data is compared to results obtained with a simulation based on the lattice Boltzmann method (LBM).
\end{abstract}

Electronic supplementary material The online version of this article (https://doi.org/10.1007/s00348-020-03014-5) contains supplementary material, which is available to authorized users.

A. Schröder

andreas.schroeder@dlr.de

$\triangle$ C. Willert

chris.willert@dlr.de

1 Deutsches Zentrum für Luft- und Raumfahrt (DLR), Institute of Aerodynamics and Flow Technology, 37075 Göttingen, Germany

2 Deutsches Zentrum für Luft- und Raumfahrt (DLR), Institute of Propulsion Technology, 51170 Köln, Germany

3 Univ. Lille, CNRS, ONERA, Arts et Metiers Institute of Technology, Centrale Lille, UMR 9014, LMFL, Laboratoire de Mécanique des fluides de Lille, Kampé de Fériet, 59000 Lille, France

4 DAAA, ONERA, University Paris Saclay, 92190 Meudon, France 


\section{Graphic abstract}

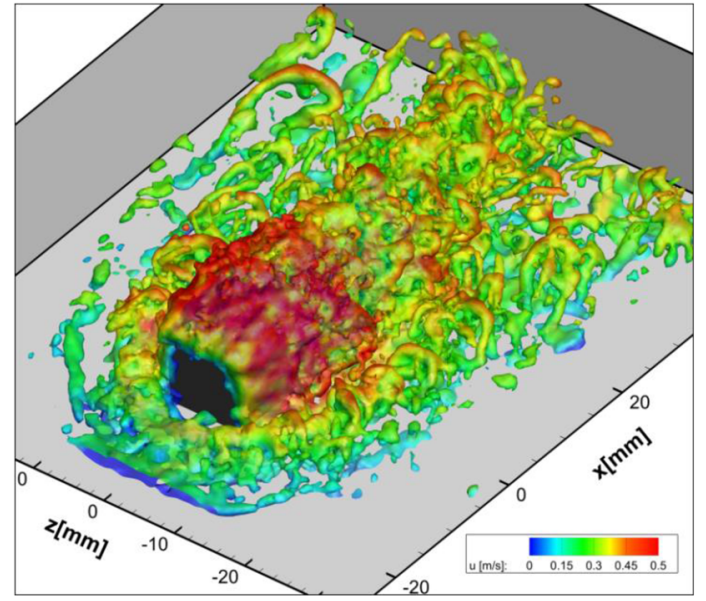

Iso-vorticity via 3D-STB and FlowFit

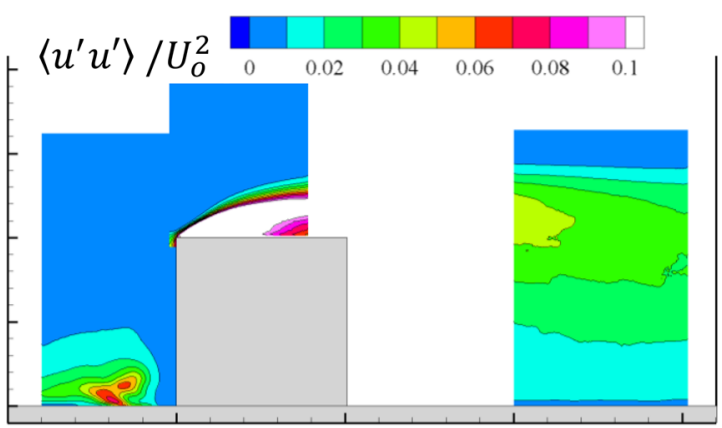

Variance of streamwise velocity obtained from time-resolved PIV (left) compared to simulation results via the Lattice-Boltzmann Method (right)
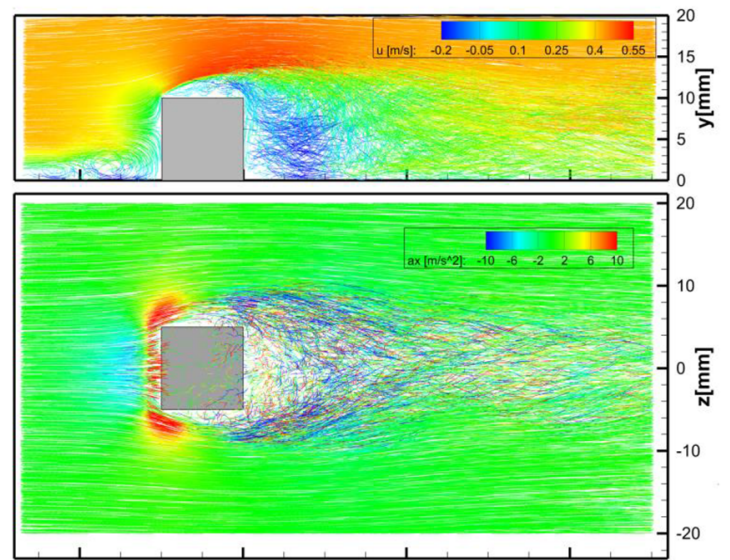

3D-STB particle tracks coded with velocity (top) and acceleration (bottom)

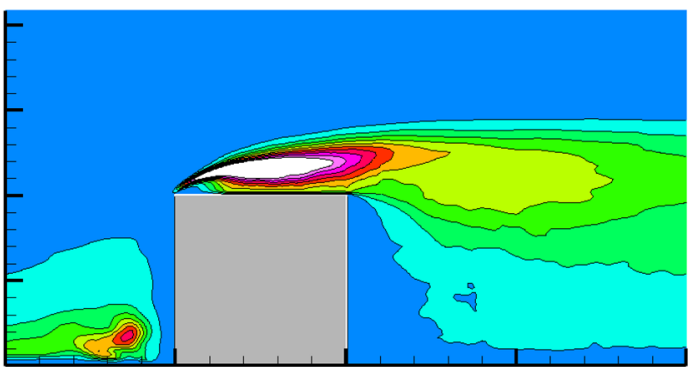

\section{Introduction}

In the last few decades, there has been extensive research on turbulent boundary layer (TBL) and turbulent channel flows around wall-attached obstacles, including cubes with various aspect ratios roughly in the range of $\delta / H \approx 0.25-5$ or $H / h \approx 0.1-0.3$. Here, $H$ is the cube or obstacle height, $\delta$ represents the boundary layer thickness and $h$ the channel height. In the first group of investigations, mainly the case with high Reynolds numbers $\left(\operatorname{Re}_{H}>10,000\right)$ and incoming TBL flows has been addressed (e.g., mimicking buildings in atmospheric BL) with the aim of identifying the general flow features around the cube. Extensive experimental studies have been performed by Castro and Robins (1977), Martinuzzi and Tropea (1993) and Meinders et al. (1999) primarily focusing on unsteady pressure distributions gained by probe measurements and wall-shear stress visualizations using oil-film techniques or similar. These studies gained many basic insights into the mean 3D flow structures around and in the wake of the cube using assumptions governed by topological concepts. Hussein and Martinuzzi (1996) generated a detailed velocity vector map in several planes and locations around the cube using one-point LDA measurements to investigate the budget balance of the turbulent kinetic energy transport by mean and fluctuation velocity statistics. Near-surface PIV results of the flat plate around and at the walls of the cube itself by Depardon et al. (2005, (2006) have allowed the estimation of the related skin friction vector fields along with the identification of locations of mean separation lines, foci- and saddle-point topologies (Depardon et al. 2007), which previously was restricted to qualitative visualizations only.

On the other hand, extensive numerical simulations using advanced methods like unsteady RANS (Iaccarino 
et al. 2003) or large eddy simulation (LES) (Krajnović and Davidson 2002; Lim et al. 2009), the latter combined with experiments) have been performed at relatively high Reynolds numbers. Direct numerical simulations (DNS) so far are limited to lower Reynolds numbers (Diaz-Daniel et al. 2017; Yakhot et al. 2006a, b) but enable a complete analysis of the unsteady behavior of 3D coherent flow structures, which in the past could be identified only partly if not even limited to mean flow structures. These structures and their characteristic dynamics depending on the governing conditions within the specific flow regions around the cube have been topic of individual research interest. Lim et al. (2007) experimentally investigated Reynolds number effects on the mean and fluctuating velocities and pressures on and around the cube for broad range of Reynolds numbers, including the investigation on a large $(6 \mathrm{~m})$ cube in an atmospheric TBL. In a subsequent study Lim et al. (2009) focused their investigations mainly on the flow, shear layers and recirculation regions above and along the three streamwise cube surfaces. In the work of Yakhot et al. (2006a), the unsteadiness of the vortical structures and induced bimodal vertical velocity distribution in front of the cube, which are the midpoints of the well-known horseshoe vortices surrounding the cube in the near wall region, was the main focus of their investigation. The extensive DNS work by Diaz-Daniel et al. (2017) of the flow around a cube, with incoming turbulent and several laminar boundary layer flows at six different low Reynolds numbers, enables the investigation of the specific dynamics of the shear layers, horse-shoe vortices and wake flows through direct comparison. They showed that the laminar in-flow condition features Reynolds number dependent specific wake dynamics with distinct peaks at several Strouhal numbers associated with the interference of transitional shear layers, the stability of the horse-shoe vortex and hair-pin vortices with the overall flow topologies of the separated flow region. This work is the closest numerical research case when compared to the parameters of the present experimental study concerning the choice of flow configurations. Although for the majority of flow cases the DNS was performed at slightly lower Reynolds numbers than in the present experimental investigation, many features quantified by the present flow measurements are very similar and can be compared directly.

In order to augment the recent DNS results with experimental data, advanced particle image based velocimetry methods were applied on a cube configuration in the water flow facility THBV $^{1}$ at ONERA, Lille. The novel dense Lagrangian particle tracking (LPT) technique ShakeThe-Box (STB) (Schanz et al. 2016) with subsequent data assimilation by FlowFit (Gesemann et al. 2016) provided

\footnotetext{
${ }^{1}$ French acronym for low speed water tunnel.
}

temporally resolved 3D3C data of the flow topology around the cube. In addition, a high resolution, time-resolved 2D2C (TR-) PIV technique (Willert 2015) provided long time-series of particle image data at selected locations to characterize the in-flow conditions and allow spectral investigations of the flow dynamics. The 3D STB and (with limitations) the TR-PIV techniques, in principle, can be adapted to flows with much higher Reynolds numbers and flow conditions that currently are beyond to reach of DNS. The employed measurement techniques are capable of very high dynamic temporal and spatial range, at least for onepoint statistics of the velocity vector data in small fields of view (Beresh et al. 2018) or by multi-pulse STB (Novara et al. 2016, 2019). For example, in compressible and swept wing aerodynamics there is continued research interest in the laminar-transitional flow conditions when the surface mounted obstacle heights $H$ are significantly smaller than the BL thicknesses, thus mimicking surface imperfections and roughness caused by limitations in manufacturing capabilities in aerodynamic vehicle production. These surface imperfections, typically in the $\mu \mathrm{m}$-range, lead to modal and non-modal growth of the induced disturbances and then to (premature) boundary layer transition during flight. The experimental investigation of transitional flow with growing small-scale disturbances in thin boundary layers around wings at transonic velocities has recently become feasible with micro 3D STB measurement methods (Novara et al. 2019). In this context, the present work can be seen as a preparatory step, taken so as to allow such laminarity studies in future research.

The collected experimental data set is also intended as a reference case for CFD validation, especially for advanced scale resolving simulation methods. In this context the Lattice Boltzmann method (LBM) has regained interest within the CFD community during the past decade since it enables time-transient simulations and gives access to instantaneous solutions of the resolved flow field. LBM is known to be capable of accurately simulating detached incompressible turbulent flows, and is therefore well suited for the turbulent flow configuration reported in this paper.

The following section describes the experimental setup of the TR-PIV and 3D STB measurement systems at the THBV water flow facility at ONERA Lille and the related specific calibration and evaluation schemes. The incoming BL flow conditions could be accurately characterized through an upstream TR-PIV measurement. Section 3 provides instantaneous and mean flow results of the TR-PIV and 3D STB measurements for a selected subset of the investigated cases. A brief description on Reynolds number effects for four laminar test cases is provided. In a next step results obtained with LBM are compared with the corresponding experimental flow field data for the turbulent test case. Further postprocessing and analysis tools applied to the collected data 


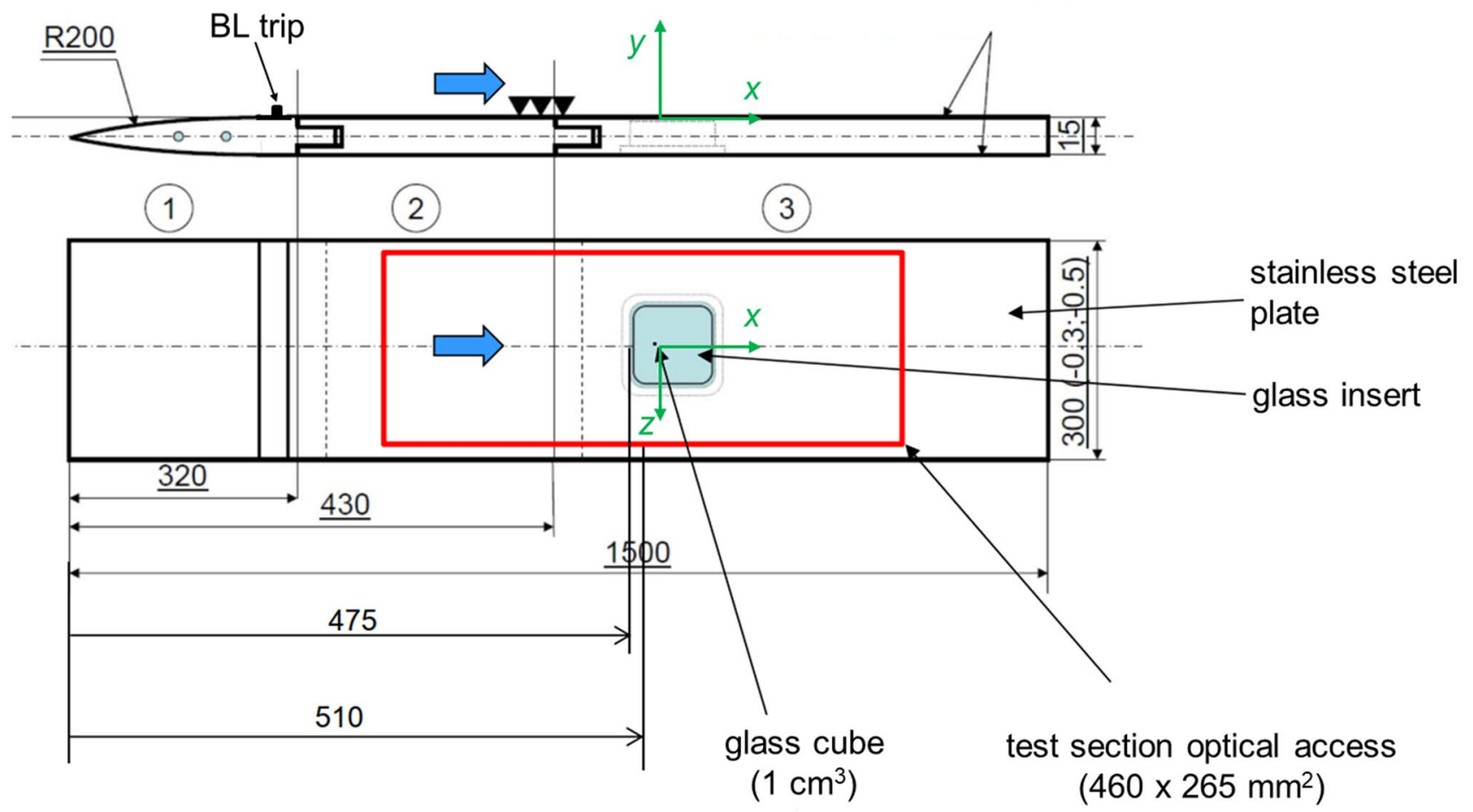

Fig. 1 Sketch of the flat splitting plate with elliptical leading edge and the location of the cube in the water tunnel

are explained and the respective findings are presented and described. The last section provides a summary of the gained results along with a short outlook.

\section{Experimental set-up and tools}

\subsection{Facility and configuration}

ONERA Lille's low speed water tunnel (THBV) is a gravity-driven, closed-loop water tunnel equipped with very good optical access, therefore making it ideally suited for particle- and laser-based velocimetry techniques. It has a square test-section of $300 \times 300 \mathrm{~mm}^{2}$ and can be operated at free-stream velocities ranging from 0.1 to $0.9 \mathrm{~m} / \mathrm{s}$. For the present test campaign, a splitting plate, with an elliptical leading edge has been placed at mid span so as to generate a boundary layer flow (see Fig. 1). In order to minimize laser light reflections on this plate, a transparent glass insert of $150 \times 150 \mathrm{~mm}^{2}$, centered in span, is placed at the streamwise position targeted for the TR-PIV and 3D STB measurements. The cube of height $H=10 \mathrm{~mm}$, also made of glass, is fixed on this plate with a refractive index matched glue and is located at a streamwise distance of $510 \mathrm{~mm}$ from the leading edge of the plate. On the premise of having a refractive index closer to that of water, different materials for the manufacture of the cube were investigated in a preliminary study, such as hydrogel based on polymerized acrylamide and PTFE (polytetrafluoroethylene or Telfon). However, none of the attempts provided a sufficiently rigid cube with defined sharp edges or sufficient transparency (e.g. for PTFE), so that glass was finally chosen. As a consequence, this necessitated specific steps in the LPT data processing to account for the refractive index variations along the imaging path as described in Sect. 2.5.

In the tests presented here, both the situations of laminar and turbulent boundary layers at thicknesses comparable to that of the cube height are considered. The TBL is triggered by a $2 \mathrm{D}$ step of $1.5 \mathrm{~mm}$ height covering the whole span that is inserted into the groove near the leading edge of the plate shown in Fig. 1, such that a low Reynolds number turbulent boundary layer is developing downstream of this tripping device.

\subsection{Measurement conditions and coordinate system}

For both 2D2C PIV and 3D STB data was acquired for the tripped, turbulent boundary layer case $U_{\infty}=0.8 \mathrm{~m} / \mathrm{s}$ at $\operatorname{Re}_{\theta}=1000$ ) and four laminar BL cases ( $U_{\infty}=0.2,0.4,0.6$ and $0.8 \mathrm{~m} / \mathrm{s}$ respectively corresponding to (rounded) $\mathrm{Re}_{H}=2000,4000,6000$ and 8000 with the cube height $H=10 \mathrm{~mm}$ and aspect ratios $\delta / H$ as indicated in Table 2 . With reference to the Reynolds numbers 

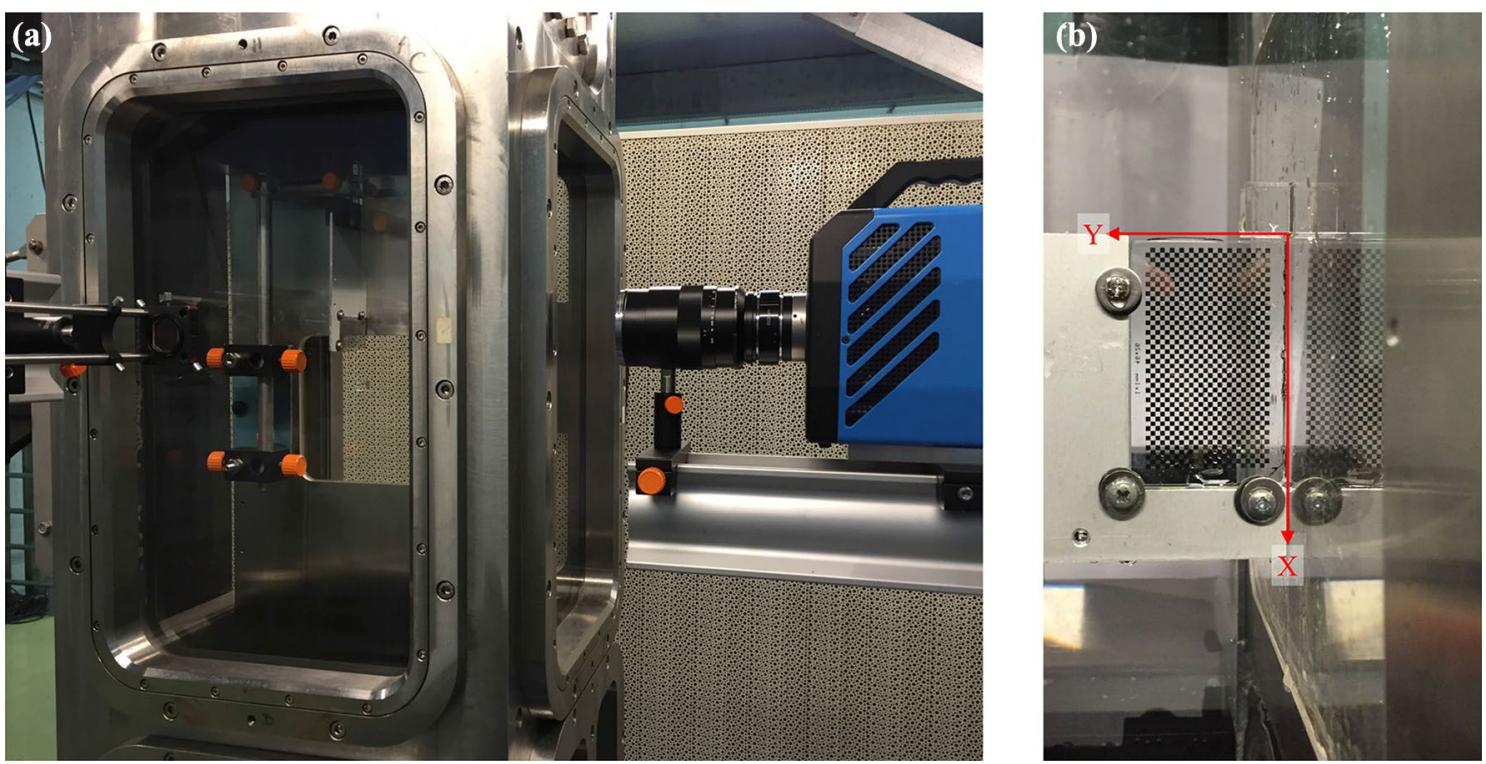

Fig. 2 High-resolution 2D-2C TR-PIV setup to image the upstream area of the cube. The calibration target can be seen installed upstream of the cube (a) and calibration target placed downstream of the cube (b). The mean flow direction in the test section is downward, along $X$

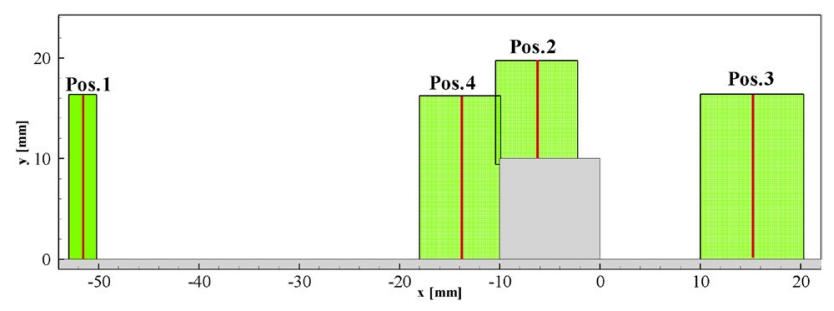

Fig. 3 TR-PIV measurement locations. Red lines indicate sample locations for spectral estimates and velocity profiles

estimated by TR-PIV upstream of the cube, we decided to round-up the values for the actual position of the cube for all further descriptions.

The coordinate system used throughout the paper is defined as follows: the plane onto which the cube is mounted is spanned by the $x$-axis, aligned in streamwise direction, and the $z$-axis in spanwise direction. Accordingly, the $y$-axis defines the wall-normal direction. The origin of the coordinate system is assigned to the base of the back face of the cube at mid-point of the spanwise dimension $z$ (see also Fig. 2b). Were appropriate length dimensions are normalized by the cube dimension $H=10 \mathrm{~mm}$.

\subsection{Time-resolved 2D-2C PIV}

The following provides an overview of the high resolution 2D-2C PIV measurements obtained along the center plane (symmetry plane, $z=0$ ) both upstream and around the cube. Measurements were performed at four locations as shown in Fig. 3. The upstream position (Pos.1) was chosen to characterize the inflow boundary layer profile upstream of the cube at $x=-52 \mathrm{~mm}(x / H=-5.2)$. Position 3 was centered at $x=15 \mathrm{~mm}$ downstream of the cube and captured the mean flow reattachment point in the wake of the cube. Position 2 mapped the area above the cube, while Position 4 sampled the stagnation zone directly upstream of the cube.

The PIV measurement approach employed in these experiments closely follows the procedures and methods laid out in the paper by Willert (2015). The near-wall measurement approach has been successfully used in a variety of previous applications, such as for turbulent boundary layer measurements with zero and adverse pressure gradients (Cuvier et al. 2017; Willert et al. 2018). The imaging setup was designed for a magnification of $m=0.66$ using a macroscopic lens of $100 \mathrm{~mm}$ focal length (Zeiss-Planar T100/2) at an aperture of $f$-number 4.0. On the imaging side a high-speed camera with a 4 megapixel CMOS sensor was used (PCO AG, DIMAX-S4), which has a pixel pitch of $11 \mu \mathrm{m}$ corresponding to $16.67 \mu \mathrm{m} / \mathrm{pixel}$ in object space (see Fig. 2 with the set-up at THBV). Using macroscopic imaging the smallest viscous scales anticipated for the turbulent case of the test matrix, corresponding to $U_{\infty} \approx 0.8 \mathrm{~m} / \mathrm{s}$ with $\operatorname{Re}_{\tau}=400$ and $\operatorname{Re}_{\theta}=1000$ amounted to about $28 \mu \mathrm{m}$ per viscous unit $\left(y^{+}=v / u_{\tau}\right)$ and could be resolved with nearly single pixel resolution.

For the upstream boundary layer measurements the field of view of the camera was reduced to 240 pixel width and 1008 pixel in height $\left(4.0 \times 16.7 \mathrm{~mm}^{2}\right)$. This reduction allowed an increase of the number of acquired images to more than 100,000 during a single run. The other roughly 5-10 $\mathrm{mm}$ wide measurement areas were illuminated by a 
pair of externally modulated continuous wave lasers (Kvant Laser, SK) with a combined output power of nearly $10 \mathrm{~W}$ at a wavelength of $520 \mathrm{~nm}$. Focusing lenses narrowed the light sheet to a thickness of about $200-300 \mu \mathrm{m}$ in the measurement area. Synchronization between modulated laser and high-speed camera was provided through a separate multichannel pulse generator (Arduino MEGA 2560).

The acquired TR-PIV image sequences were processed using a 2D-2C PIV processing algorithm based on cross-correlation, using a coarse-to-fine pyramid approach with image deformation and intermediate validation (normalized median filter and smoothing). To achieve optimal, noise-minimized velocity estimates, a pyramid correlation scheme, similar to the algorithm proposed by Lynch and Scarano (2013), was employed and used up to five consecutive frames. For the upstream measurement position (Pos.1 in Fig. 3) a large aspect ratio of $64 \times 3$ pixels $\left(1070 \times 50 \mu \mathrm{m}^{2}\right)$ was chosen to achieve optimal wall-normal spatial resolution in the recovered velocity profiles. In wall-normal distance the sampling distance was fixed at 1 pixel $(16.7, \mu \mathrm{m}$. The data from all other measurement locations (Pos.2, 3 and 4) were processed with a final interrogation window size of $16 \times 16$ pixels $\left(270 \times 270 \mu \mathrm{m}^{2}\right)$. Sampling distances were approximately $30 \%$ of the sample size.

\subsection{D STB experimental set-up}

The flow around the cube was also investigated with the time-resolved 3D STB technique enabling dense Lagrangian particle tracking in an extended volume around the cube. In total seven high-speed cameras and a high repetition laser for volume illumination (see Figs. 4 and 5) were used to carry out the experiments. Measurements were performed at the same in-flow conditions as for the TR-PIV measurements reported in Sect. 2.3. Corresponding acquisition parameters for each point of the test matrix are given in Table 1. Both flow cases are of eminent interest for the understanding of this complex geometry induced flow separation case with transitional and turbulent shear and wake flow development regions.

A hexagonal prism was installed at the front glass window of the water tunnel to realize nearly orthogonal viewing conditions through the air-glass-water interfaces for each camera avoiding strong astigmatism effects for particle imaging. In order to capture the flow in the measurement volume around the cube in full time-resolution, a system of seven high-speed cameras (6× PCO.Dimax S4 with 4 MP
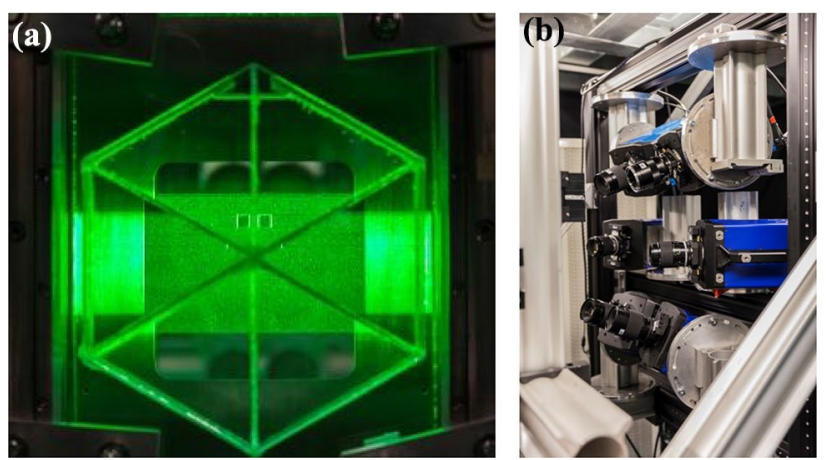

Fig. 4 Laser volume illumination of particles in the flow around the surface mounted glass cube as viewed through six-folded prism (a). Six high-speed cameras pointed at the measurement volume (b). A seventh camera viewed the cube from the opposite side

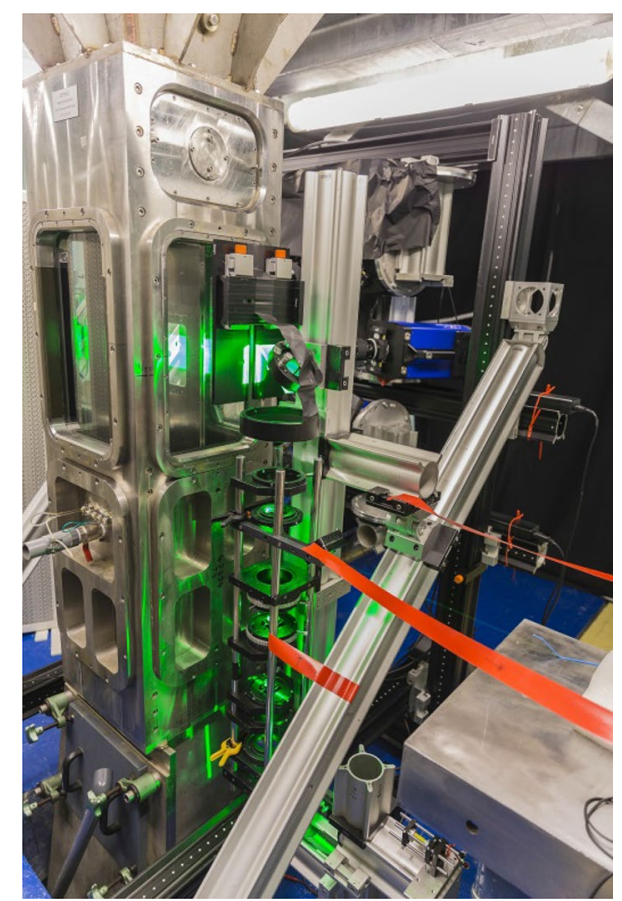

Fig. 5 Photograph of the experimental setup with light-sheet optics attached to the tunnel and camera system in the back

with $11 \mu \mathrm{m}$ pixels and $1 \times$ Phantom Miro 340 with $10 \mu \mathrm{m}$ pixels reduced to $\approx 2 \mathrm{MP}$ ) was set up. The six $\mathrm{PCO}$ cameras obliquely viewed the prism with nearly the same relative viewing angles through the prism, which itself was attached to the glass window of the water tunnel with a glycerol-water
Table 1 Measurement matrix for the volumetric 3D STB experiment

\begin{tabular}{lllllll}
\hline Condition & \multicolumn{3}{c}{ Laminar } & & Turbulent \\
\hline Tunnel setting, $U_{\infty}$ & {$[\mathrm{m} / \mathrm{s}]$} & 0.8 & 0.6 & 0.4 & 0.2 & 0.8 \\
Acquisition rate & {$[\mathrm{Hz}]$} & 1500 & 1125 & 750 & 375 & 1500 \\
Total image count & & 29,400 & 22,050 & 22,044 & 22,044 & 55,125 \\
\hline
\end{tabular}



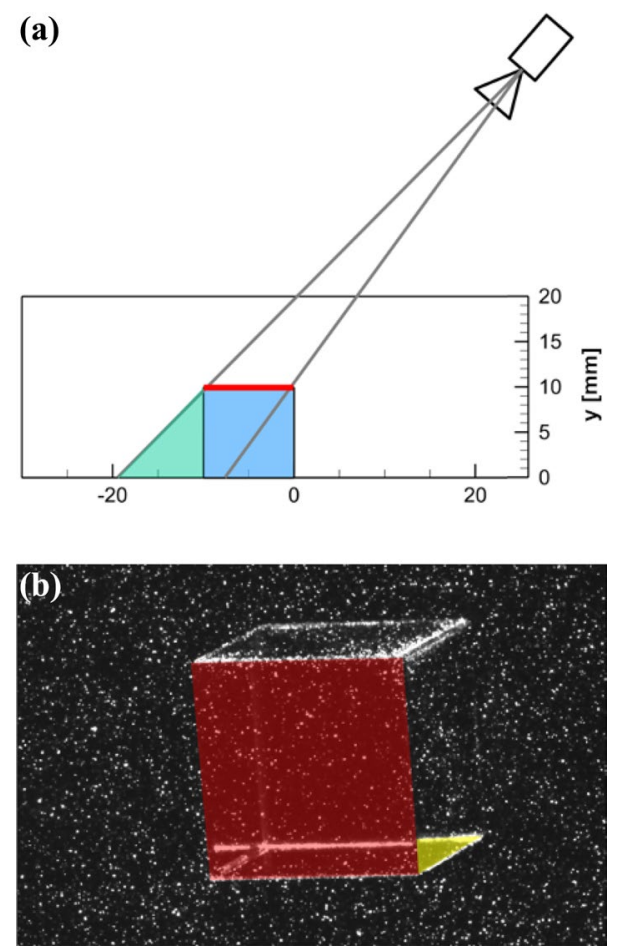

Fig. 6 a Single camera viewing the cube, with indicated 'shadow' area (green), where particle images would appear displaced. b Representative particle image of the cube as viewed by the camera. A 2D mask applied on cube's top is indicated in red

mixture as a thin adhesive fluid layer in between the two parallel glass surfaces of the prism and water tunnel, respectively. The seventh camera (Phantom Miro) viewed through the window on the opposite side and was set to a highmagnification factor to image only the area surrounding the cube. This camera acquired complementary images of the "shadow" regions induced by refraction through the cube on the images of the other six cameras. A high-repetition laser system (Quantronix Darwin Duo) with an energy of $20 \mathrm{~mJ}$ per pulse at $1 \mathrm{kHz}$ was used to illuminate a rectangular section of approximately $80 \mathrm{~mm}$ in streamwise direction and $20 \mathrm{~mm}$ in wall-normal direction using beam shaping optics and a passe-partout to cut off the weaker intensity of the expanded laser beam. In addition the collimated laser beam was back-reflected through the measurement volume by a mirror (see Figs. 4 and 5). The common field of view of the six camera system covered a section of approximately $70 \mathrm{~mm}$ in spanwise direction. The six PCO cameras were equipped with $100 \mathrm{~mm}$ focal length lenses (Zeiss MakroPlanar T 100/2) while the Phantom camera was equipped with a 200 mm lens (Nikon Micro Nikkor 200/4).

\subsection{Challenges in STB particle tracking around the cube}

For the specific challenges faced during the calibration of the 3D STB system and subsequent evaluation of particle images several improvements on top of the existing time-resolved Lagrangian particle tracking code have been implemented and tested. First the calibration and volume matching of the seventh camera view with a much smaller field of view and higher magnification factor has been adapted. Starting with the calibration target the number of visible markers in the seventh camera was too low in order to realize a common volumetric calibration together with the common view of the other six cameras. Therefore, as first indicator, the eight cube edges were detected manually in the field of view of the seventh camera. As their 3D positions are known, they were used as preliminary 3D-2D point correspondences. This calibration was refined by tracking results that were gained using only the other six cameras: the tracked particles located in the common view with the seventh camera were back-projected into this camera using the preliminary calibration, which was accurate enough to identify the corresponding peaks in the original camera image. The particle coordinates and the identified corresponding peak locations constitute a huge number of accurate $3 \mathrm{D}-2 \mathrm{D}$ point correspondences, allowing for a precise calibration of the seventh camera. Then for all cameras, a local determination of the particles' optical transfer function (OTF) were estimated according to Schanz et al. (2012), following the volume self calibration step (Wieneke 2008).

In a second challenge, a volumetric mask was created in order to take into account that the cube is located within the measurement volume and thus within the individual cameras lines-of-sight. Although the cube was made of glass (necessary for the volumetric, collimated and back-reflected laser illumination), its refractive index ( $n=1.520)$ is sufficiently different from the refractive index of water $(n=1.333)$. Due to this difference in refractive index the images of particles located in the 'shadow' of the cube for a specific camera (the green area in Fig. 6a) will undergo a significant shift, as they are viewed through the cube glass surfaces under different angles. This displacement would corrupt the reconstructed 3D position, therefore the cameras seeing the particle through the cube glass need to be ignored in the shaking process.

Due to the simple geometry of the cube, the projection points of individual particles, combined with a constraint on their height above the wall, can be used to isolate particles that are viewed through the cube for each camera. As a 'mask' we will define the image of the top surface of the cube for a given camera (see Fig. $6 b$ for one exemplary camera view). It should be noted that no image processing is performed with this mask; it purely defines a search 
area. Figure 6a illustrates that particles which are projected onto this mask for a given camera could be located in three domains. Particles located above the cube are visible without disturbance by all (Depardon et al. 2006) camera views, therefore all cameras can be used for the shaking procedure. Particles located in the shadow region behind the cube are displaced and have to be excluded from the reconstruction process for the given camera view. A particle could also be (falsely) reconstructed within the cube, however this rarely occurs. In this case the current camera is also ignored. Therefore, the condition whether a given camera view should be included in the shaking process for a given particle is dependent on the particle location [(above the cube $(y>10 \mathrm{~mm})$ or below the cube $(y<10 \mathrm{~mm})]$ and on its projection point (within the mask or not). Despite leaving out multiple cameras in some regions, it was possible to view all areas around the cube with at least four cameras due to the chosen $6+1$ camera configuration with one camera viewing the cube orthogonally from the opposite side. Particles below $y=10 \mathrm{~mm}$ and a projection point within the yellow area could either be located in front of the cube or in the small shadow region on the side. It was decided to not apply a mask in this region as this would have resulted in some (small) regions being reconstructed by less than four cameras.

The polyamide seeding particles sourced from Vestosint introduced an unfavorably high dynamic range of intensities accompanied by a variation of the particle image diameters between two and eight pixels. The utilized particles have a polydisperse distribution with a large variation of shapes and diameters between $\approx 5 \mu \mathrm{m}$ and $80 \mu \mathrm{m}$ and an average diameter of $\approx 30 \mu \mathrm{m}$. For future investigations in water facilities similar to the present we plan to use particles that are less polydisperse, such as, for instance, low-cost polyamide particles (e.g., Orgasol). STB evaluation was further complicated by the fact that the flow is locally highly turbulent and three-dimensional with very strong shear regions near the geometry-induced flow separations starting at the front edges of the cube. Near the strong shear layers a predictor from neighboring particles was not reliable. Furthermore, particles are subjected to strong acceleration events while passing around the edges of the cube or move within the turbulent wake of the cube which resulted in a reduction in accuracy of their predicted position for the following time step and subsequent loss of some tracks in these regions. In part, this is due to limitations of the temporal resolution.

In addition, high intensities in the background image from the light scattered at the cube edges further complicated the evaluation situation of particle tracks close to the edge positions. To overcome some of the difficulties related to these regions and high acceleration events we used averaged 3D flow field results from preliminary STB evaluations. Results from the evaluation of one run per flow case were binned in
3D bins of $1 \mathrm{~mm}$ edge length (akin to the method introduces in Sect. 3.4). The collected 3D velocity averages in each of these small sub-volumes are then used as a predictor field of particle position $\mathbf{p}$. The search radius around a point predicted by this field is then parameterized by the local RMS value determined in the binning process. Depending on the particle location this RMS-value can be very small (e.g., in a region far away from the cube) or quite large (e.g., in the shear layers close to a cube edge). In order not to reject true events, the following relation was used for the RMS-based search radius: $\sigma_{\mathrm{RMS}}=7 * \mathrm{RMS}+0.5$ pixel. The locally varying expression $\mathbf{p}+\sigma_{\mathrm{RMS}}$ is used throughout the complete volume both for the identification of new tracks within the clouds of newly reconstructed particles and as an outlier criterion for already tracked particles. On average around 250-400 particles (about $0.8 \%$ of the particles) are excluded per time-step by this criterion. These additional schemes were included in the STB evaluation to enable a robust and reliable 3D Lagrangian particle tracking in the full volume.

\subsection{FlowFit: Navier-Stokes regularized data assimilation to a Cartesian coordinate system}

After fitting the found particle tracks temporally by thirdorder B-splines with an optimized parametrization from spectral analysis (TrackFit by Gesemann et al. 2016), we gain improved particle positions, velocities and accelerations in a scattered manner within our 3D measurement volume by analytical differentiation. To get the full time-resolved 3D velocity fields and the related velocity, gradient tensor- and pressure fields on (any) Cartesian grid, we further calculate a data assimilation of the scattered particle information using a continuous flow field representation by fitting a system of uniform third order cubic B-splines to the given scattered discrete velocity and acceleration vectors gained from the particle tracks. The FlowFit data assimilation scheme applies a fully incompressible Navier-Stokes regularization which is imposed onto the grid of B-splines by optimizing iteratively a set of related cost functions using a non-linear optimization algorithm (here LBFGS). The related cost functions are weighted according to the measurement noise and are penalizing strong curvature, divergence of velocity and of Eulerian acceleration together with the differences between measurements and interpolations at particle locations (Gesemann et al. 2016). FlowFit avoids the classical correlation-window-based low-pass filtering (a common short-coming of tomographic PIV), increases the spatial resolution by following the imposed physical constraints and allows for an arbitrary sampling on desired grids as well for derivations which can be directly computed on any position in the measurement volume from the continuous B-spline functions (e.g., vorticity, strain, Q-criterion and similar quantities). 


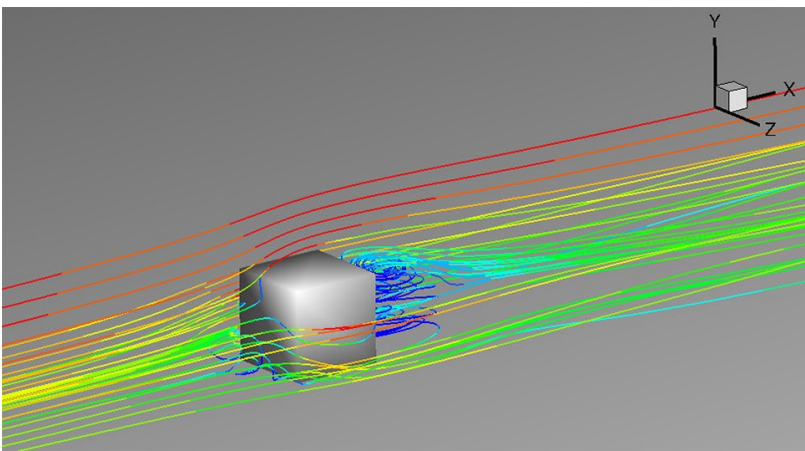

Fig. 7 Mean flow field around the cube from the LBM simulation for the turbulent test case at $U_{\infty}=0.8 \mathrm{~m} / \mathrm{s}$

\subsection{LBM methodology}

The flow around the cube is simulated using the lattice Boltzmann flow solver ProLB. This solver is based on the lattice Boltzmann method (Qian et al. 1992; Chen and Doolen 1998) that is implemented on a non-uniform octree grid (Touil et al. 2014). Briefly outlined, the LBM is based on a particle collision model in the Lagrangian frame of reference. It can be interpreted as a discrete version of the Boltzmann equations that govern the gas motion, represented at the mesoscopic level by a probability density function of a reduced number of velocity vectors. The LBM is thus a sequence of propagation and collision of fictitious particles. The ability of LBM to simulate bluff body flows at different Reynolds numbers has already been demonstrated by Liu et al. (2008) and Islam et al. (2012). The present simulation is based on ProLB, a novel CFD solver intended to address low-Mach number flows around highly complex geometries. The volume mesh is a multi-resolution Cartesian grid that is generated automatically. Complex solid objects are treated using an immersed solid boundary algorithm. The turbulence modeling is based on a large eddy simulation (LES) approach with a shear improved Smagorinsky model. A wall model is added to take into account near-wall turbulence effects and adverse pressure gradients (Tannoury et al. 2014). In this study the flow condition with incoming turbulent boundary layer with $U_{\infty}=0.8 \mathrm{~m} / \mathrm{s}$ is considered.

For the volumetric grid refinement, the ProLB solver requires a Cartesian grid with embedded resolution domains. Special care is given to ensure sufficient distance between two consecutive resolution domains to allow a correct propagation of the velocity distribution functions at the nodes. The simulation domain counts a total of seven resolution domains corresponding to $11 \times 10^{6}$ equivalent fine voxels in the volume. In terms of initial conditions, a constant velocity profile is set at the inlet of the simulation domain, while a pressure release boundary condition is used at the outlet. A no-slip boundary condition is set on all the walls (flat
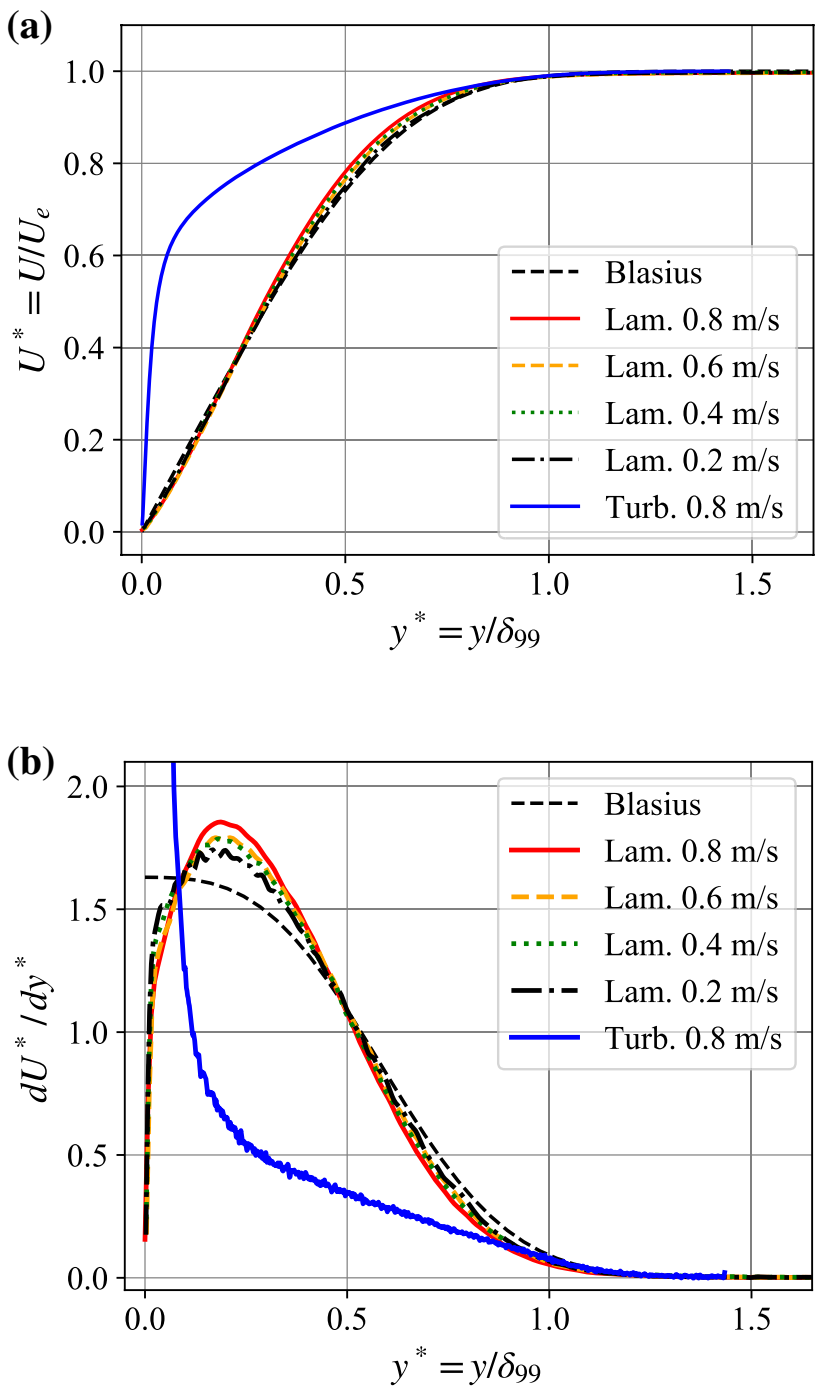

Fig. 8 a Mean velocity profiles at $x=-52 \mathrm{~mm}$ upstream of the cube scaled with outer velocity $U_{e}$ and boundary layer thickness $\delta_{99}$. b Profiles of the mean normalized velocity gradient $\mathrm{d} U^{*} / \mathrm{d} y^{*}$

plate, cube, tunnel walls) and the floor of the flat plate is treated with an artificial roughness to ensure a proper development of the incoming turbulent boundary layer. The inlet velocity profile has been adjusted accordingly to match the experimental velocity profile, both in magnitude and fluctuations, that is captured upstream of the cube at $x=-52.0 \mathrm{~mm}$ $(x / H=-5.2)$. The convergence is monitored using probes located near the inlet and upstream and downstream the cube. The fluid measurement files are averaged over the last 200,000 iterations, corresponding to an average over $57.7 \mathrm{~s}$ physical time. A typical time-averaged flow field is provided in Fig. 7. It exhibits the flow structures that are discussed in the next section in comparison to the experimental results. 

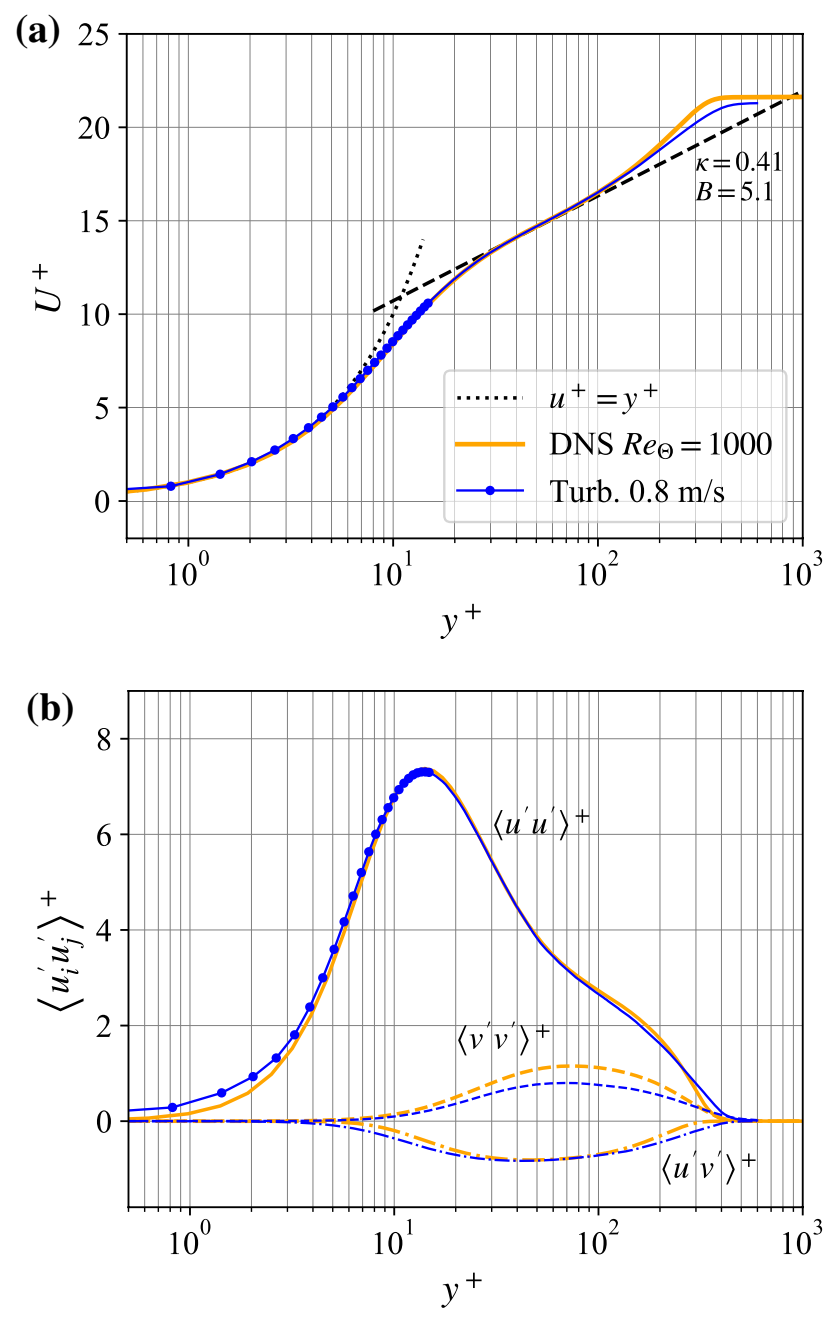

Fig. 9 a Velocity profile for turbulent case at $x=-52 \mathrm{~mm}$ upstream of the cube normalized by inner variables (viscous scaling). b Corresponding variances and co-variance of streamwise and wall-normal velocity components scaled with inner variables. Orange set of lines are from DNS data by Schlatter et al. (2009). For clarity symbols are only shown for $y<20^{+}$

\section{Results and discussion}

\subsection{Characterization of the upstream flow}

As described in Sect. 2.3, the flow conditions upstream of the cube were characterized using 2D-2C TR-PIV sequences acquired at $2 \mathrm{kHz}$ with a duration of nearly one minute (107,540 images). In order to normalize the time-averaged velocity profiles, estimates of the wall shear stress estimates were obtained through fits to DNS data (for the turbulent case) or using theoretical solution (e.g., Blasius profile for the laminar cases). Additional single pixel-line processing of the image data, using the methodology proposed in Willert (2015), yielded a further estimate of the wall shear rate (and shear stress). The relevant boundary layer parameters determined for the upstream position at $x=-52 \mathrm{~mm}$ are summarized in Table 2 for all measurement conditions.

Compared to the best-fit to the DNS data performed in the range $10^{+}<y<200^{+}$, the wall-shear rate estimate from single pixel-line processing is underestimated by about $5 \%$ which can be attributed to a bias of the estimated near wall velocity toward zero. More advanced processing algorithms such as 2D STB are required here to properly reject erroneous near-wall measurement data (for a comparison of 2D-2C TR-PIV with 2D STB techniques see Schröder et al. 2018).

The mean velocity profiles obtained from the image sequences are presented in Fig. 8a for both the laminar and turbulent cases. Normalization is based on the outer velocity $U_{e}$ and the 99-percentile boundary layer thickness $\delta_{99}$. Figure $8 \mathrm{~b}$ presents profiles of the normalized velocity gradient and highlights the difference of the measured laminar velocity profiles to the Blasius solution. Owing to the normalization, the integral of the velocity gradient profiles is unity for the Blasius solution and approaches 1.0 to within a percent for the measurements (see Table 2) and is a quality indicator of the overall velocity profile. Noteworthy is the presence of a peak in the velocity gradient at $y \approx 0.2 \delta_{99}$ for all investigated laminar flow conditions. While not investigated further, the observed deviation from the Blasius profile may originate from the elliptic profile at the leading edge of the flat plate onto which the cube is mounted.

The turbulent velocity profile, normalized with inner (viscous) variables, is presented in Fig. 9a together with DNS data provided by Schlatter et al. (2009) for a Reynolds number of $\operatorname{Re}_{\theta}=1000$. Corresponding profiles of the fluctuation of the streamwise velocity $\left\langle u^{\prime} u^{\prime}\right\rangle^{+}$, the wall-normal velocity $\left\langle v^{\prime} v^{\prime}\right\rangle^{+}$and covariances $\left\langle u^{\prime} v^{\prime}\right\rangle^{+}$are shown in Fig. 9b. For the most part, the measurement data for the turbulent case agrees well with the DNS data, except for the wall-normal fluctuations $\left\langle v^{\prime} v^{\prime}\right\rangle^{+}$that tend to be underestimated. This could be attributed to spatial smoothing by the rather large length of the correlation window size in flow direction of 64 pixel corresponding to $1.07 \mathrm{~mm}$ or $39^{+}$. Also the rather low dynamic range of the wall-normal velocity component with a maximum standard deviation of $\approx 0.9$ pixel can be affected by pixel locking effects (Christensen 2004).

Spatially resolved power spectral densities (PSD) of the incoming velocity profiles are provided in Fig. 10. All quantities are normalized using the incoming velocity $U_{\infty}$ and the cube size $H$ to allow a better comparison between the different flow conditions. The normalized frequency, defined as $\hat{f}=f H U_{\infty}^{-1}$, is equivalent to the Strouhal number St. To better highlight small variations across the frequency range, a pre-multiplication of the normalized spectral power $\hat{\phi}$ with the normalized frequency (i.e., Strouhal number $\mathrm{St}$ ) attenuates the typically higher PSD levels at lower frequencies while amplifying signals at the upper frequencies. The PSD for the turbulent in-flow indicates a maximum energy 
Table 2 Characteristic parameters of the boundary layer at upstream position, $x=-52 \mathrm{~mm}$ (Pos.1)

\begin{tabular}{|c|c|c|c|c|c|c|}
\hline \multirow{2}{*}{$\begin{array}{l}\text { Condition } \\
\text { Reynolds number } \operatorname{Re}_{H}\end{array}$} & & \multirow{2}{*}{$\begin{array}{l}\text { Turb. } \\
8000\end{array}$} & \multicolumn{4}{|l|}{ Laminar } \\
\hline & & & 8000 & 6000 & 4000 & 2000 \\
\hline Tunnel setting $U_{\infty}$ & {$[\mathrm{m} / \mathrm{s}]$} & 0.8 & 0.8 & 0.6 & 0.4 & 0.2 \\
\hline Measured $U_{e}$ & {$[\mathrm{~m} / \mathrm{s}]$} & 0.829 & 0.831 & 0.629 & 0.419 & 0.208 \\
\hline BL thickness, $\delta_{99}$ & {$[\mathrm{~mm}]$} & 11.29 & 4.36 & 4.81 & 5.77 & 7.39 \\
\hline$\delta / H$ & - & 1.13 & 0.44 & 0.48 & 0.58 & 0.74 \\
\hline Displacement thickness, $\delta^{*}$ & {$[\mathrm{~mm}]$} & 1.79 & 1.46 & 1.63 & 1.90 & 2.55 \\
\hline Momentum thickness, $\theta$ & {$[\mathrm{mm}]$} & 1.24 & 0.56 & 0.62 & 0.72 & 0.97 \\
\hline Shape factor ${ }^{1}, \delta^{*} / \theta$ & - & 1.45 & 2.61 & 2.63 & 2.61 & 2.64 \\
\hline Friction coefficient, $c_{f}$ & - & 0.00417 & 0.00111 & 0.00130 & 0.00165 & 0.00242 \\
\hline Mean wall shear rate, $d U /\left.d y\right|_{y=0}$ & {$\left[\mathrm{~s}^{-1}\right]$} & 1394 & 363 & 242 & 136 & 49.7 \\
\hline Estimated wall shear rate ${ }^{2}$ & {$\left[\mathrm{~s}^{-1}\right]$} & 1430 & 319 & 220 & 124 & 45.9 \\
\hline Shear rate integral, $\int_{0}^{\infty}\left(d U^{*} / d y^{*}\right) d y *$ & - & 0.978 & 0.994 & 0.993 & 0.995 & 0.994 \\
\hline Estimated friction velocity, $u_{\tau}$ & {$[\mathrm{m} / \mathrm{s}]$} & 0.0389 & 0.0196 & 0.0160 & 0.0120 & 0.0073 \\
\hline Viscous length, $y^{+}=v / u_{\tau}$ & {$[\mu \mathrm{m}]$} & 27.2 & 54.0 & 66.2 & 88.3 & 146 \\
\hline Viscous length in image space & [pixel] & 1.6 & 3.3 & 4.0 & 5.3 & 8.8 \\
\hline$R e_{H}=U_{\infty} H / v$ & - & 7820 & 7840 & 5930 & 3950 & 1970 \\
\hline$R e_{\delta}=U_{\infty} \delta_{99} / v$ & - & 8830 & 3320 & 2850 & 2280 & 1460 \\
\hline$R e_{\theta}=U_{\infty} \theta / v$ & - & 996 & 438 & 368 & 288 & 190 \\
\hline$R e_{\tau}=U_{\tau} \delta_{99} / v$ & - & 415 & 81 & 73 & 65 & 51 \\
\hline
\end{tabular}

${ }^{1}$ Shape factor for Blasius profile: $\delta^{*} / \theta=2.59$.

${ }^{2}$ Wall shear rate estimates for laminar cases are based on fit to Blasius solution about $0.5 \mathrm{~mm}$ from the wall, near the inner turbulence peak ( $y \approx 15^{+}$or $0.4 \mathrm{~mm}$ ), which is in agreement with the body of literature. The spectral power gradually decreases throughout the vertical extent of the boundary layer. For the laminar in-flow condition, the flow, for the most part, is free of significant PSD levels, aside from a low frequency peak at $\hat{f} \approx 0.01(0.8 \mathrm{~Hz})$ at a wall distance of $y \approx 2 \mathrm{~mm} \approx 0.4 \delta_{99}$. This low-frequency power does not seem to influence the flow around the cube as it cannot be detected in any of the spectra presented in the results section (see Sect. 3.2). The source of this spectral power is believed to be a low frequency modulation which might stem from water tunnel pumping and/or structure vibration eigenfrequencies with its respective receptivities of the laminar boundary layer. The signal is present at the same position for all but the lowest laminar Reynolds number with $U_{\infty}=0.2 \mathrm{~m} / \mathrm{s}$.

\subsection{TR-PIV for 2D-2C velocity fields}

The following figures provide information of the flow around the cube exemplified for the case at $U_{\infty}=0.8 \mathrm{~m} / \mathrm{s}$ which allows a comparison between experiment and LBM simulation. In Fig. 11 interesting flow features can be observed: For the turbulent flow case corresponding to $\delta / H=1.12$ the mean reattachment point downstream of the flow separation in the cube wake is located at $x \approx 15 \mathrm{~mm}(x / H=1.5)$, while it shifts further downstream to $x \approx 18.5 \mathrm{~mm}(x / H=1.85)$ for the laminar condition with $\delta / H=0.44$. Diaz-Daniel et al. (2017) provided the closest DNS with respect to present conditions and they gave a reattachment point value for the turbulent case at $x / H=1.5$ and a stagnation point value slightly above the wall for the laminar case at $x / H=0.93$. For the turbulent case the value agrees very well with our finding despite the difference in the Reynolds numbers $\left(\operatorname{Re}_{H}=3000\right.$ for DNS compared to 8000 for the experiment). Aside from the Reynolds number difference, the lower ratio of $\delta / H=0.44$ for the present laminar case also might contribute to a more extended mean recirculation bubble of up to $x / H=1.85$ compared to the DNS which is based on a ratio of $\delta / H=1$. On the other hand, for the present laminar case at $\operatorname{Re}_{H}=2000$ with a corresponding $\delta / H=0.74$, the reattachment point shifts upstream to $x / H=1.2$ (see Fig. 22) which is closer to the DNS result provided at $\operatorname{Re}_{H}=3000$. Furthermore, the vertical extent of the separation bubble on top of the cube is larger for the laminar case which is caused by the larger deflection angle of the incoming flow at the upstream upper edge of the cube due to the narrower laminar boundary layer thickness.

Between the laminar and turbulent cases, the distribution of the turbulent kinetic energy (TKE) differs significantly. Whereas the TKE is typically defined as one half of the sum of the three velocity variances $\left\langle u_{i}^{\prime} u_{i}^{\prime}\right\rangle$, the present TR-PIV measurements only provide variances for the two in-plane velocity components $u$ and $v$. The missing variance for the 
(a)
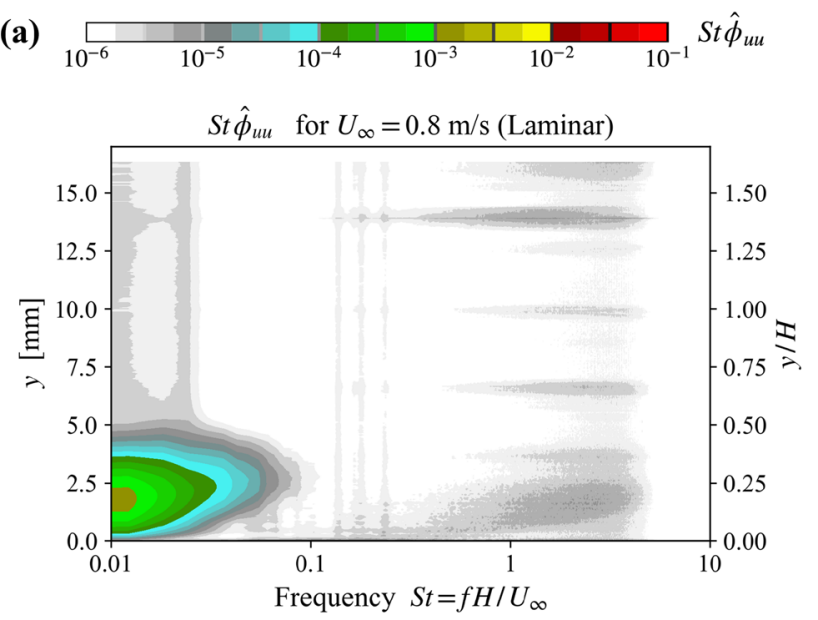

(b)

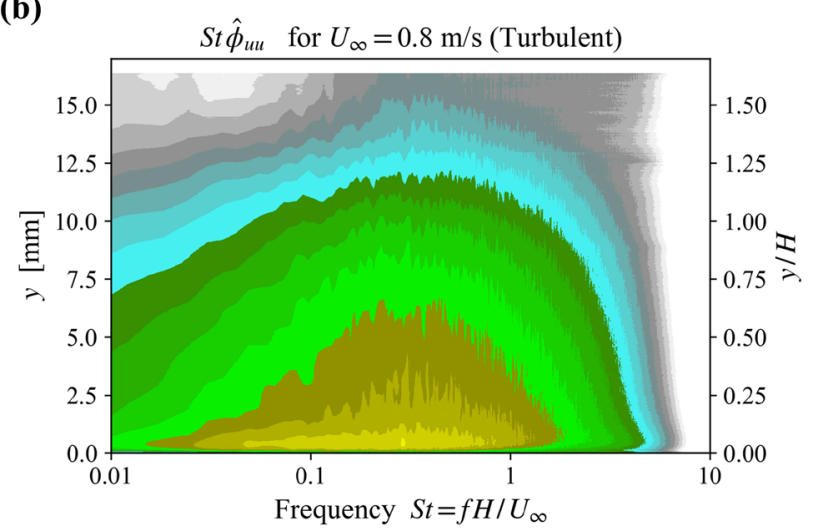

Fig. 10 Pre-multiplied frequency spectra of the streamwise velocity profile at $x=-52 \mathrm{~mm}$ upstream of cube for the laminar (a) and turbulent in-flow condition (b)

out-of-plane component $\left\langle w^{\prime} w^{\prime}\right\rangle$ is estimated as the mean of the measured variances such that the TKE is approximated by $\frac{3}{4}\left(\left\langle u^{\prime} u^{\prime}\right\rangle+\left\langle v^{\prime} v^{\prime}\right\rangle\right)$. The turbulent flow case exhibits larger values of the (TKE) in the cube's shear layer on top of the flow separation, whereas they are reduced almost by one half in the wake region compared to the laminar condition. A distinct point of high TKE is visible upstream of the cube close to the wall for the turbulent case which can be identified as the point upstream of the first-order horse-shoe vortex origin. Here a dynamic bi-modal vertical velocity PDF has been previously documented by Yakhot et al. (2006a).

The differences between the TKE values in the cube wake for the two boundary layer flow types can be visualized as well by the two corresponding time traces of the streamwise and wall-normal velocity components at the position of the mean reattachment point for the turbulent case near $x / H=1.5$. Here, a higher dynamics of streamwise velocity and specifically of small scale wall-normal velocity events with strong positive and negative values can be recognized for the laminar in-flow condition shown in Fig. 13. For the turbulent in-flow condition corresponding time traces, provided in Fig. 14, the wake dynamics are less pronounced and spatially and temporally smoother (Fig. 12). Note that the differences of the data resolution between the time traces of the incoming laminar (Fig. 13) and turbulent (Fig. 14) flow case is caused by the lower temporal resolution of the laminar TR-PIV measurements.

The mean flow field resulting from the LBM simulation of the turbulent case are provided in Fig. 11, bottom row. The simulation results exhibit a very similar distribution structure for both velocity components and the TKE in comparison to the corresponding time resolved PIV result (Fig. 11, middle row). This agreement can also be seen in the streamlines in Fig. 25 where the different structures are well reproduced in size and position for the horseshoe vortex upstream of the cube, for the recirculation bubble on the top face and in the wake. While the TKE distribution obtained from the measurements relies on an approximation using only two velocity components, Fig. 12 provides an additional side-by-side view of the velocity variances of the in-plane velocity components (i.e., $u$ and $v$ ) obtained from experiment and simulation. This also confirms that the chosen TKE approximation is viable for this configuration along the symmetry plane at $z=0$.

The influence of the in-flow conditions on the wake dynamics can also be observed in the power spectra obtained along a wall-normal line at $x=15 \mathrm{~mm}$. Figure 15 provides normalized power spectra of the streamwise velocity for the two conditions, pre-multiplied by the non-dimensional frequency St to more clearly highlight differences. Noteworthy, is a pronounced signal for the laminar condition at a nondimensional frequency of $\mathrm{St} \approx 0.1$ which extends all the way to the wall. This frequency previously has been documented in literature for a cube in turbulent channel flow (Meinders et al. 1999; Yakhot et al. 2006b) and is associated with periodic large-scale vortex shedding in the cube's wake. Unlike the findings reported in the literature, the experimental data for the turbulent in-flow condition does not exhibit this pronounced frequency and rather has a much more broad-band power spectral density distribution peaking at $\mathrm{St} \approx 0.5$ and $y \approx 11 \mathrm{~mm}(y / H \approx 1.1)$. Spatially this spectral density peak coincides with the peak TKE for both laminar and turbulent in-flow. On the other hand it is noteworthy that turbulent boundary layer and turbulent channel flows lead to various differences in the reported dynamics (Castro and Robins 1977; Yakhot et al. 2006b) such that the discrepancies observed here are not surprising.

\subsection{Time-resolved 3D-3C velocity and acceleration fields}

Evaluation of the complete volumetric data set given in Table 1 was performed successfully using Lagrangian 

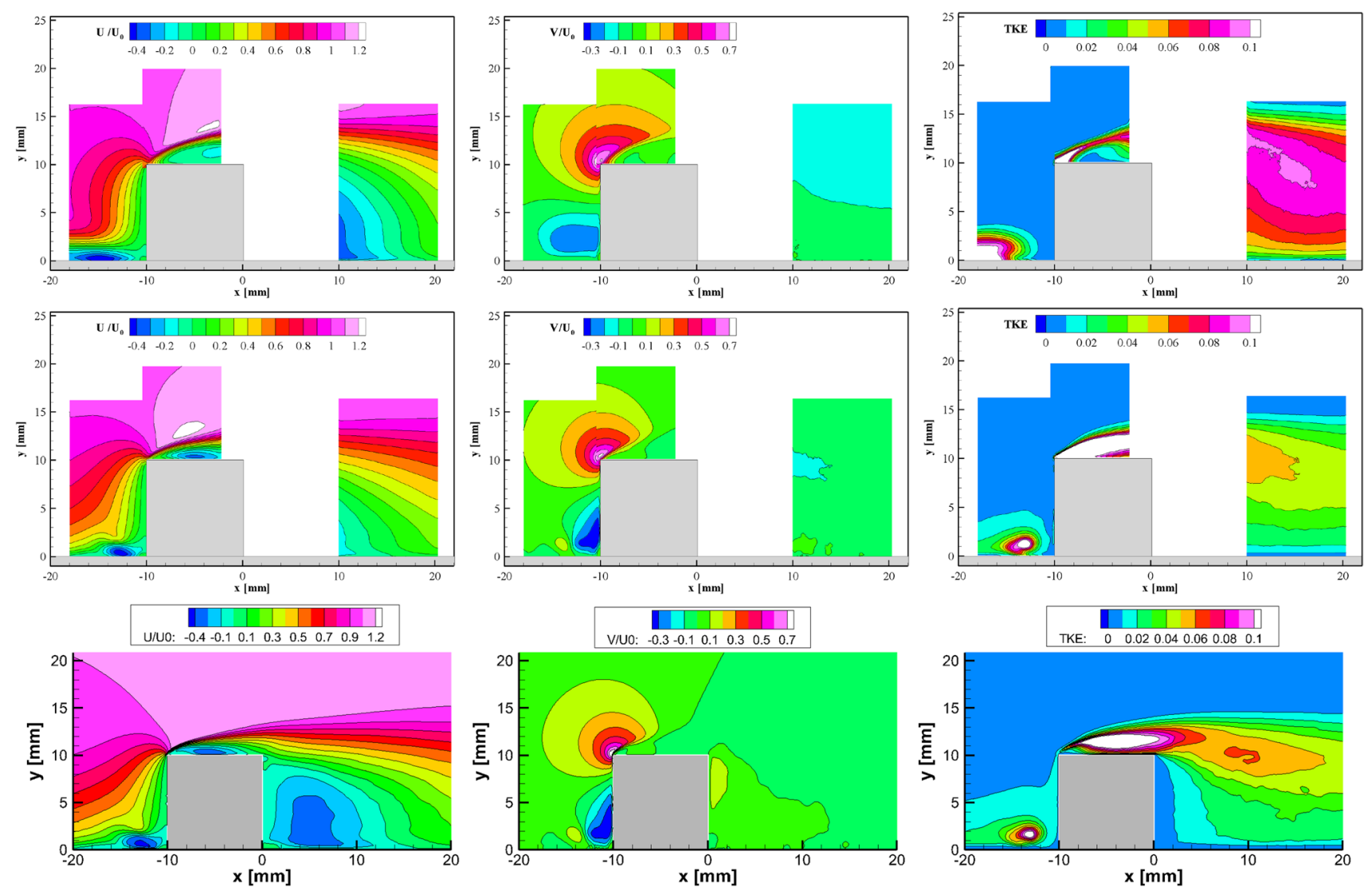

Fig. 11 Flow fields around the cube at center plane $(z=0)$ obtained with TR-PIV for the laminar in-flow case (top row) and turbulent inflow case (middle row). Bottom row shows corresponding flow fields obtained with LBM for the turbulent case. Mean streamwise velocity (left column), mean wall-normal velocity (middle column) and turbulent kinetic energy (right column), all normalized by the in-coming flow velocity $U_{\infty}=0.8 \mathrm{~m} / \mathrm{s}$ particle tracking (LPT) by the Shake-The-Box (STB) algorithm (Schanz et al. 2016). Figures. 16 and 17 show results for a case with laminar in-flow conditions at $U_{\infty}=0.4 \mathrm{~m} / \mathrm{s}$. Within the reconstructed volume around 70,000 particles were instantaneously tracked over many time steps and the velocity and acceleration resp. material derivative information was computed directly from the continuous temporal B-spline fit function (TrackFit). For this case 22,050 successive images at a recording frequency of $750 \mathrm{~Hz}$ are available (see Table 1). Figure 16, top, shows particle tracks in proximity of the wall at $0<y<1.2 \mathrm{~mm}$ are color coded by streamwise velocity. On the right of Fig. 16 at $U_{\infty}=0.2 \mathrm{~m} / \mathrm{s}$ tracks are shown for a slightly larger wall parallel slice color coded by wall normal acceleration, which nicely highlights the horse shoe vortices present in front and on both sides of the cube close to the wall.

Measuring Lagrangian acceleration by STB a dense distribution enhances the quality of the FlowFit data assimilation scheme: On the left hand side of the imposed Navier-Stokes momentum equation. These input values directly allow the calculation of accurate instantaneous 3D pressure distributions (Huhn et al. 2018). The upper left of Fig. 17 shows a side view of a central slice at $z=0$ with $3 \mathrm{~mm}$ thickness comprising 60 successive time-steps. Here, the motion of particles approaching the cube within the incoming boundary layer of the flow can be observed. The particles are being decelerated and deflected by the adverse pressure gradient field in front of the cube (see Fig. 26) and then are either transported over the cube when arriving above the stagnation point on the cube's front surface or towards the wall if below. Particles deflected downward either move into the horseshoe vortex and then around the cube as indicated in Fig. 16, bottom, or when pushed even closer to the wall, they tend to travel in an upstream direction within a thin layer.

Many particles remain close to the wall and travel around the cube at a surprisingly large radius and low velocity (see Fig. 16). Large, elongated vortices are visualized by the trajectories, bent around the cube (see Figs. 16 and 17 right). Separation bubbles are present at the three streamwise faces of the cube (best visible in Fig. 17 left up for the top face), where fluid flows backwards, until the vortex structures are 

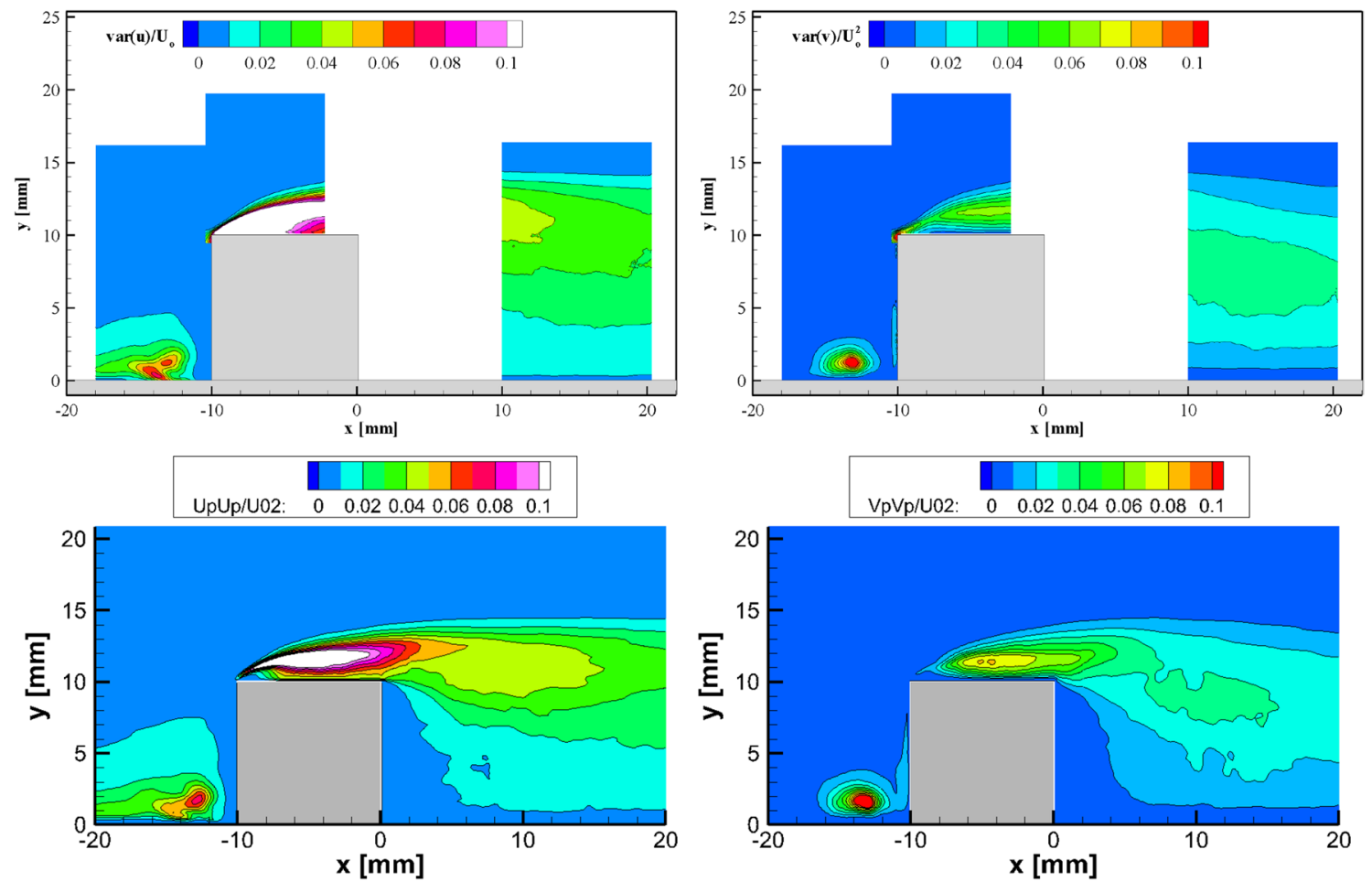

Fig. 12 Comparison between TR-PIV (top row) and LBM results (bottom row) for the velocity variances: streamwise velocity (left column) and wall-normal velocity (right column) at center plane $(z=0)$, all normalized by the in-coming flow velocity $U_{\infty}=0.8 \mathrm{~m} / \mathrm{s}$

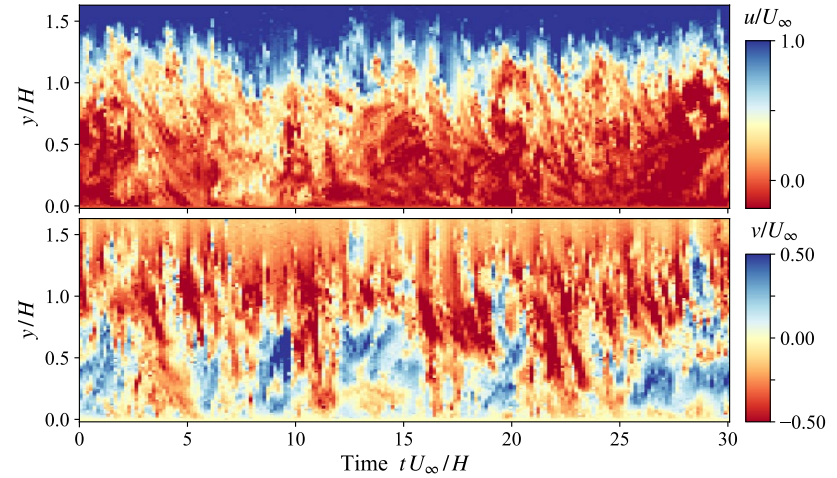

Fig. 13 Portion of the velocity profile time record for the laminar condition downstream of the cube at $x / H=1.5$ (Pos.3) at $U_{\infty}=0.8 \mathrm{~m} / \mathrm{s}$ showing streamwise velocity (top), wall-normal velocity (bottom), both normalized by the incoming bulk velocity $U_{\infty}$

carried away by the suction along the sharp shear layer that is created at the edges of the cube. Here, the highest accelerations are seen (see Fig. 17, bottom left). Strongly turbulent flow is generated in the wake of the cube specifically for the higher laminar BL flow velocities.

Using the FlowFit algorithm, the densely tracked particles can be used in conjunction with physical regularizations to interpolate the velocity, acceleration and pressure field onto a regular grid. As a result the temporally resolved

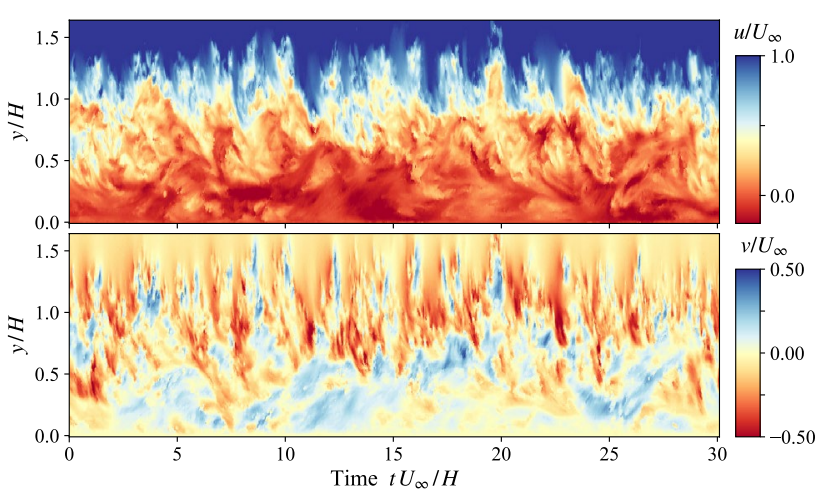

Fig. 14 Portion of the velocity profile time record for the turbulent condition downstream of the cube at $x / H=1.5$ (Pos.3) showing streamwise velocity (top), wall-normal velocity (bottom), both normalized by the incoming bulk velocity $U_{\infty}$

3D velocity and acceleration gradient tensor is available. Figure 17, right shows an instantaneous FlowFit result with iso-surfaces of vorticity. The cube is covered by connected regions of shear vorticity that quickly break up into vortices of different scales, as the flow becomes turbulent. The bent vortices surrounding the cube in upstream direction that were already visible in Fig. 17, right can now be clearly identified. A large horseshoe vortex, surrounding the lower part of the cube that breaks up when secondary hairpin-type 
(a)
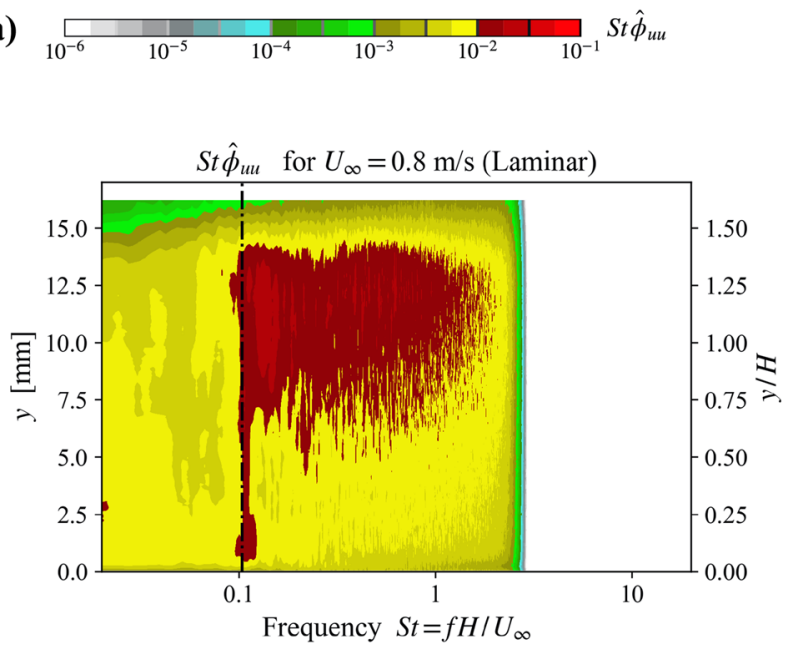

(b)

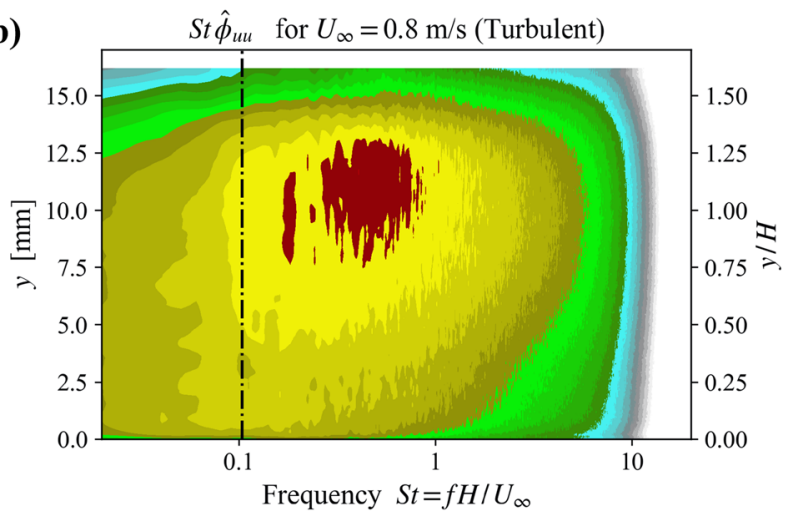

Fig. 15 Pre-multiplied spectra of the normalized streamwise velocity $u$ at $x / H=1.5$ in the wake of the cube for laminar BL in-flow (a) and turbulent BL in-flow (b), both at $U_{\infty}=0.8 \mathrm{~m} / \mathrm{s}$. The dashed line represents $\mathrm{St}=0.104$ as reported in literature

vortices are developed, is visible. Two other flow field examples visualized by $3 \mathrm{D}$ vorticity iso-contour surfaces of the laminar case with $U_{\infty}=0.8 \mathrm{~m} / \mathrm{s}$ are given in Fig. 18 .

In Fig. 18, the influence of the cube on the stability of the laminar flow is clearly visible along the generated shear layers and the horse-shoe vortices. In all related shear flow regions first typical Kelvin-Helmholtz instabilities generate waviness leading to the rapid evolution of hairpin-like vortices. The downstream development of similar vortex structures on top of the horseshoe vortices is as well visible on both sides of the cube. The hairpin-like vortices evolve in wall-normal and spanwise direction and are convected downstream inducing entrainment events through the wake shear layer and a spanwise growing of a turbulent wedge.

The higher wake dynamics of the laminar in-flow condition in comparison to the turbulent case can also be observed in the spectra presented in Fig. 15. For the laminar case at $U_{\infty}=0.8 \mathrm{~m} / \mathrm{s}$ a strong signal is present at $S t=\hat{f} \approx 0.1$ which (a)

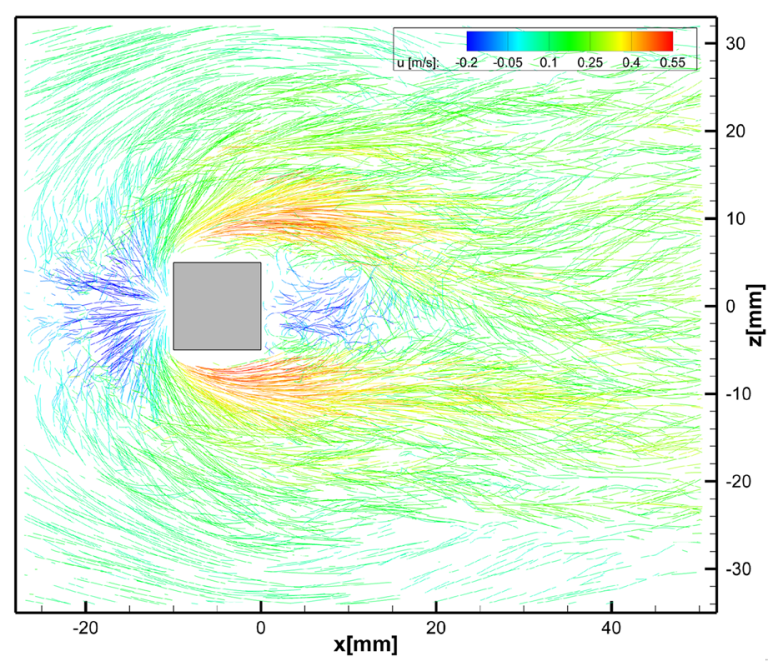

(b)

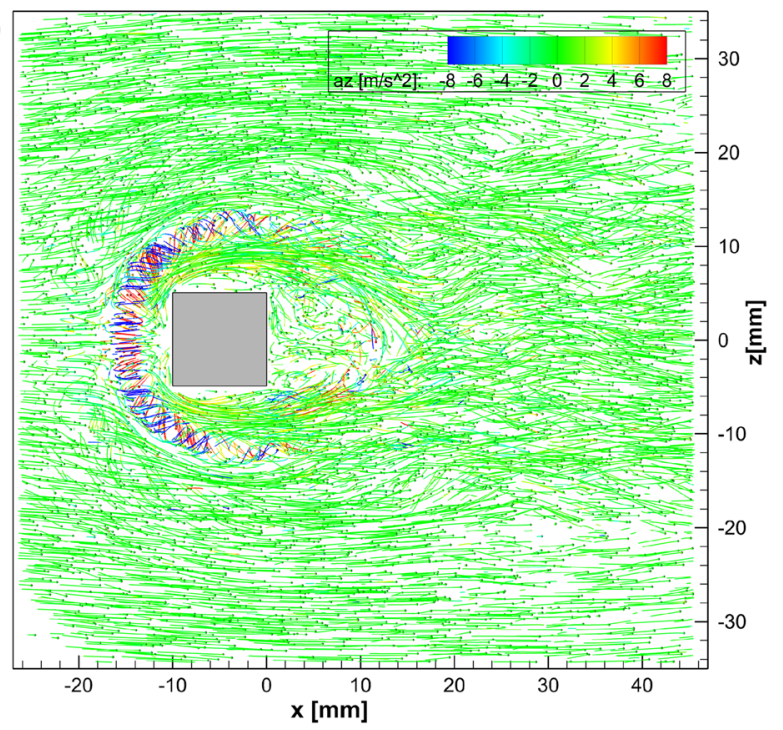

Fig. 16 a Particle tracking results for $U_{\infty}=0.4 \mathrm{~m} / \mathrm{s}$ laminar in-flow, displaying a slice close to the wall $(y=0-1.2 \mathrm{~mm})$ color-coded by streamwise velocity. b $3 \mathrm{D}-\mathrm{STB}$ result obtained at laminar in-flow of $U_{\infty}=0.2 \mathrm{~m} / \mathrm{s}$ with slice through volume at $y=0-3.5 \mathrm{~mm}$ with color coding of the wall-normal acceleration. Animated versions of these figures are available in the supplementary material

can be attributed to large scale vortex shedding from the cube and can be observed all the way to wall. This value is consistent with the value of $\mathrm{St}=0.104$ reported in the literature (Yakhot et al. 2006b; Meinders et al. 1999). For the turbulent case, the vortex shedding is much less pronounced and shifted toward higher frequencies, while the overall PSD is roughly reduced by a factor of 2 . Closer to the wall the wake dynamics of the laminar case are also more pronounced in comparison to the turbulent condition which agrees with the visual impression obtained from Figs. 13 and 14 . 
(a)

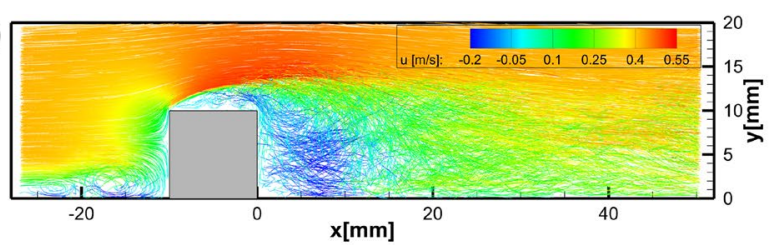

(b)

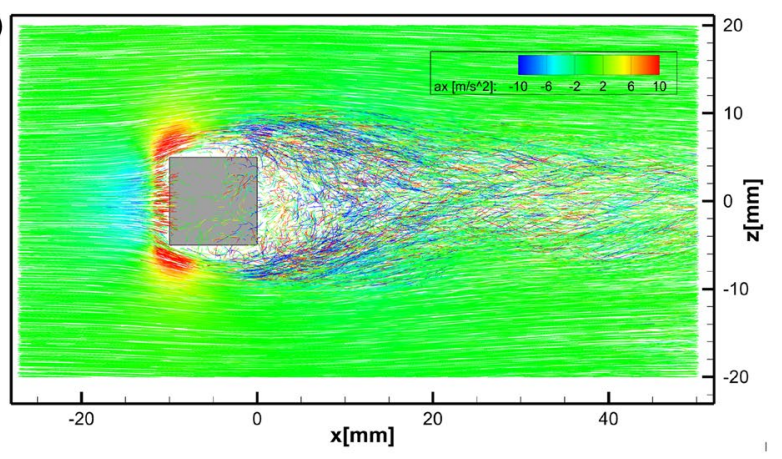

(c)

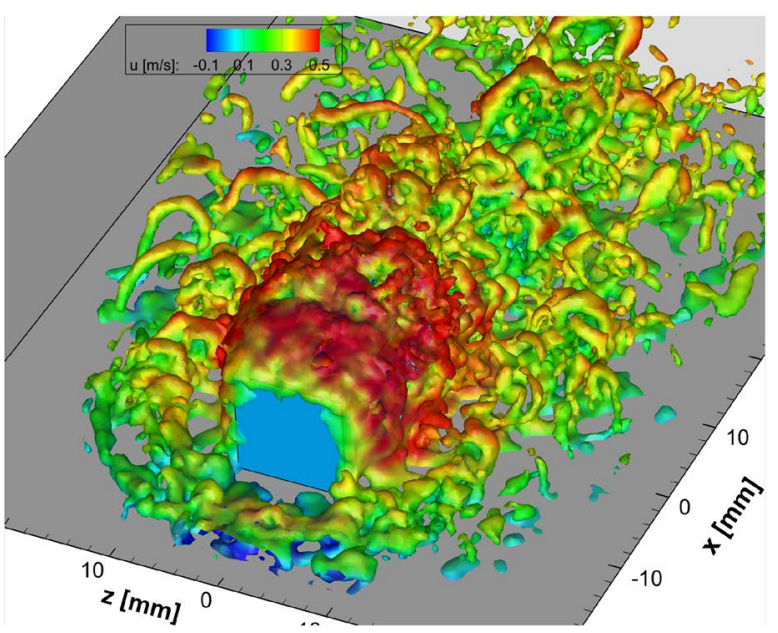

Fig. 17 3D STB result for $U_{\infty}=0.4 \mathrm{~m} / \mathrm{s}$ laminar in-flow. a Side view on a $3 \mathrm{~mm}$ slice at the mid plane of the cube. Particle tracks of 60 successive time-steps, color-coded by streamwise velocity. b View from above on a $1.3 \mathrm{~mm}$ slice near the top of the cube $(y=$ 9.5-10.8 mm), color-coded by streamwise acceleration. c Instantaneous snapshot of vorticity iso-surfaces $\left(|\omega|=170 \mathrm{~s}^{-1}\right)$ color coded by u-velocity, as calculated by FlowFit from the STB tracking results. An animated version of the iso-vorticity rendering is available in the supplementary material (a)

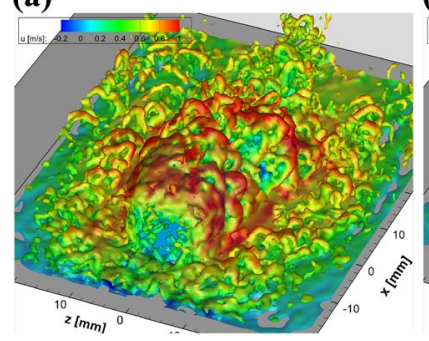

(b)

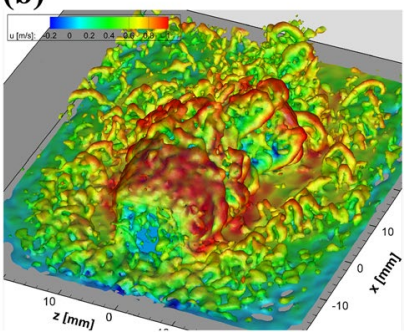

Fig. 18 Two successive time-steps of $U_{\infty}=0.8 \mathrm{~m} / \mathrm{s}$ laminar case with vorticity iso-surfaces by FlowFit
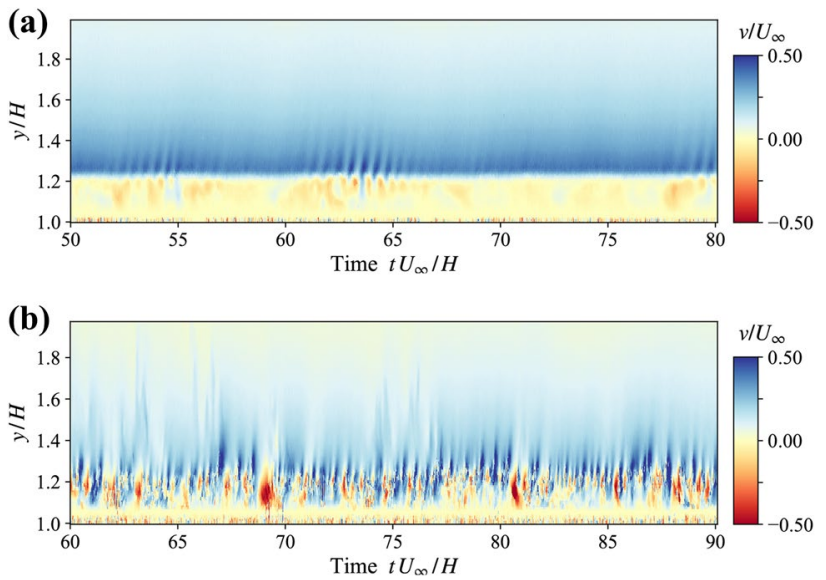

Fig. 19 Portion of the velocity time record of the wall-normal velocity above the cube at $x / H=-0.4$ (Pos.2) for the laminar condition (top) at $U_{\infty}=0.4 \mathrm{~m} / \mathrm{s}$ and turbulent condition at $U_{\infty}=0.8 \mathrm{~m} / \mathrm{s}$ (bottom). Both plots are normalized by the incoming bulk velocity $U_{\infty}$

(a)
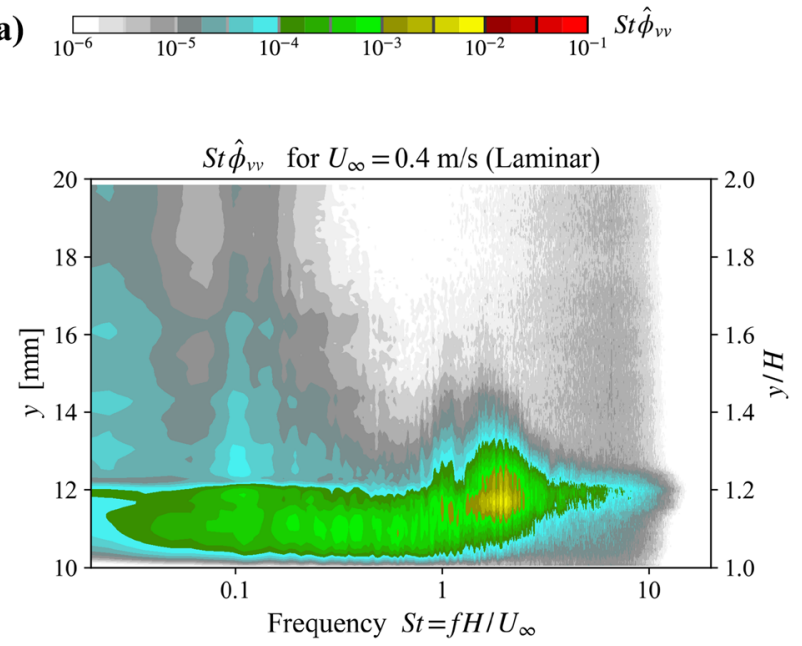

(b)

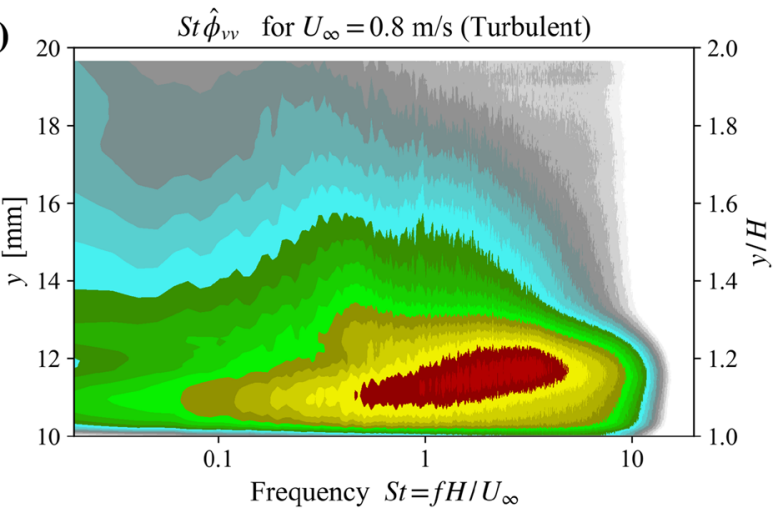

Fig. 20 Pre-multiplied spectra of the wall-normal velocity above to cube at $x / H=-0.4$ for laminar BL in-flow (a) and turbulent BL inflow (b) 


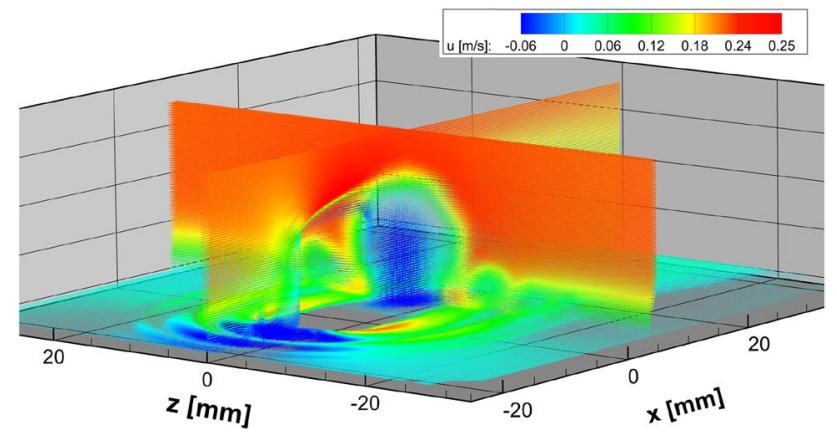

Fig. 21 Result of 3D mean velocity field for the laminar BL flow case at $U_{\infty}=0.2 \mathrm{~m} / \mathrm{s}$ based on bin-averaging approach and visualized by three perpendicular vector planes color coded by the streamwise velocity. Data set consists of nearly $10^{7}$ volume bins of size $0.25^{3} \mathrm{~mm}^{3}$ (a)

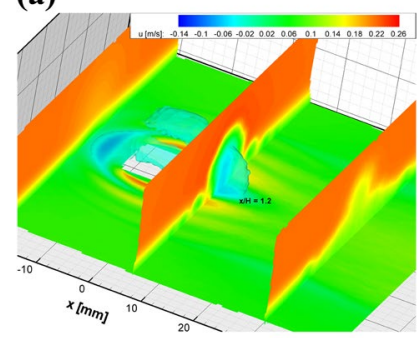

(c)

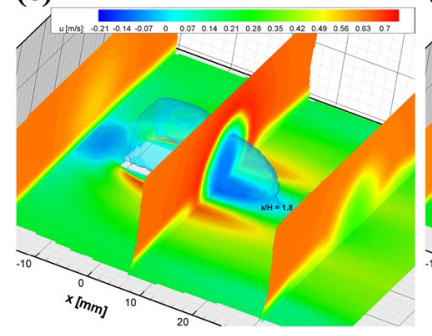

(b)

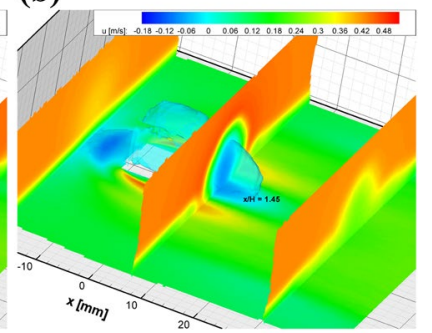

(d)

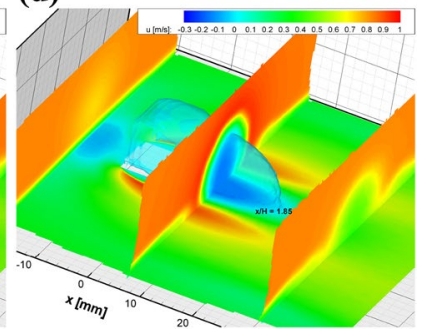

Fig. 22 Bin averaged 3D velocity fields of the laminar test cases at in reading order increasing $U_{\infty}=[0.2 ; 0.4 ; 0.6 ; 0.8] \mathrm{m} / \mathrm{s}$ and corresponding $\operatorname{Re}_{H}=[2000 ; 4000 ; 6000 ; 8000]$. Color coded streamwise velocities $u$ at four extracted planes, with the horizontal plane at $y=1 \mathrm{~mm}$ above the wall. Iso-contour surfaces enclose negative $u$-velocity values. Note the differences in the color bar

Turning attention to the shear layer above the cube, the flow exhibits considerable differences between laminar and turbulent in-flow conditions. For the turbulent condition the shear layer dynamics are very random as testified by both the velocity time record (Fig. 19, bottom) and the associated broad-band frequency spectrum (Fig. 20, bottom). The laminar in-flow, on the other hand, exhibits a shear layer with a non-periodic transition to short-lived, fine-scale vortex shedding whose signature can be seen in vertical velocity component of the time record in Fig. 19, top. The frequency of this intermittent shedding peaks at $\mathrm{St} \approx 2$ and is more than (a)

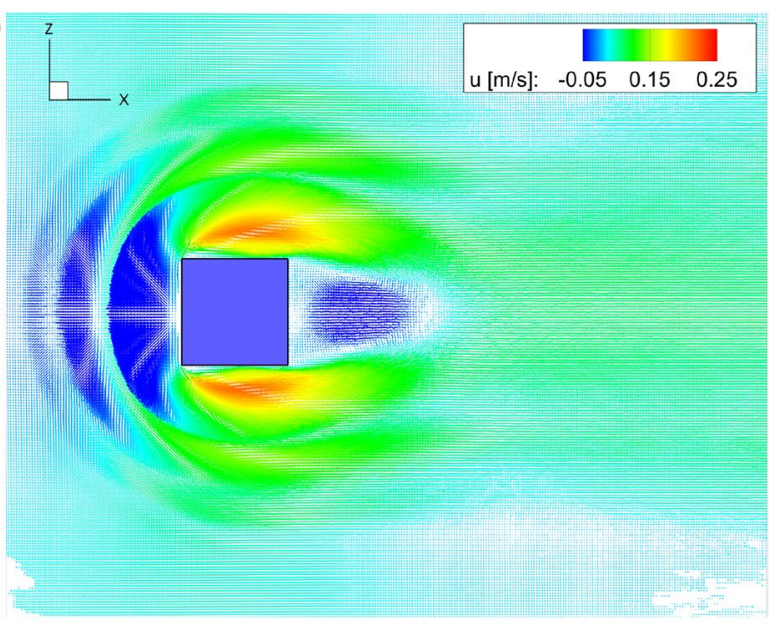

(b)

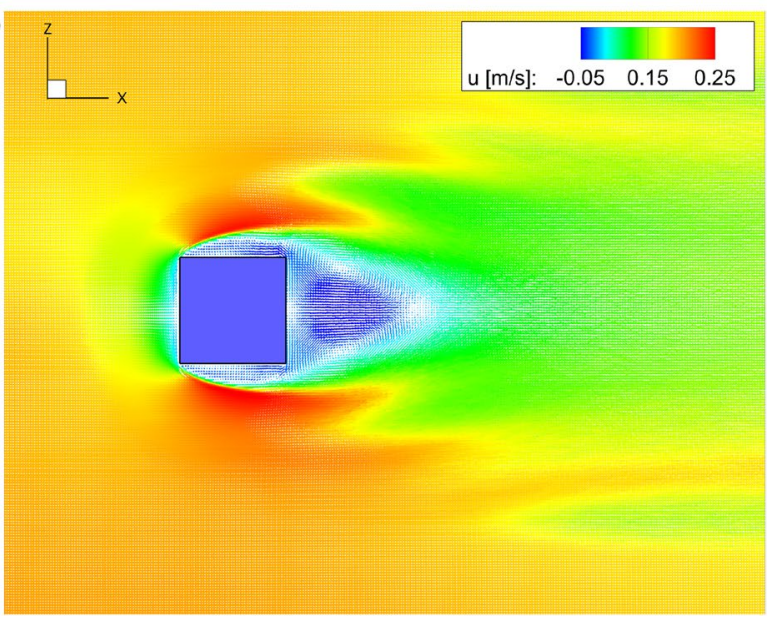

Fig. 23 Result of 3D mean velocity field for laminar boundary layer flow case at $U_{\infty}=0.2 \mathrm{~m} / \mathrm{s}$ based on bin-averaging approach (see Fig. 21) and visualized by a vector plane at $y=0.5 \mathrm{~mm}$ (indicative of the wall shear stress distribution) (a) and at $y=5 \mathrm{~mm}$ (b), both color coded by the streamwise velocity component

an order of magnitude higher than the large scale shedding of $\mathrm{St} \approx 0.1$ observed in the wake of the cube.

\subsection{D bin-averaging results}

A straight-forward statistical approach which preserves the high spatial resolution of the available particle tracking data while being adaptable to the threshold requirements of statistical convergence of the different one- or multi-point statistics is the so called "bin-averaging" method. Here the complete data set of about 22,000 time steps of the tracking results are used to build a dense grid of $9.85 \times 10^{6}$ bins with an individual cubic bin size of $0.25^{3} \mathrm{~mm}^{3}$. A result of the converged mean flow field color coded by the streamwise velocity is given in Fig. 21 and shows a volumetric overview of the mean flow field by three orthogonal slices through the domain. It represents a small part of the maximal possible 
(a)

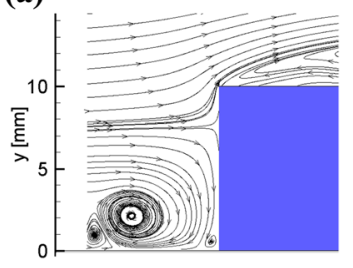

(b)

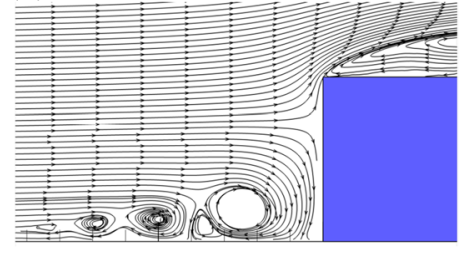

Fig. 24 Mean velocity streamlines from TR-PIV measurements for laminar boundary layer flow case at $U_{\infty}=0.2 \mathrm{~m} / \mathrm{s}$ at $z=0 \mathrm{~mm}$ (a) and corresponding streamlines cut of 3D STB bin-averaging result (b)

(a)
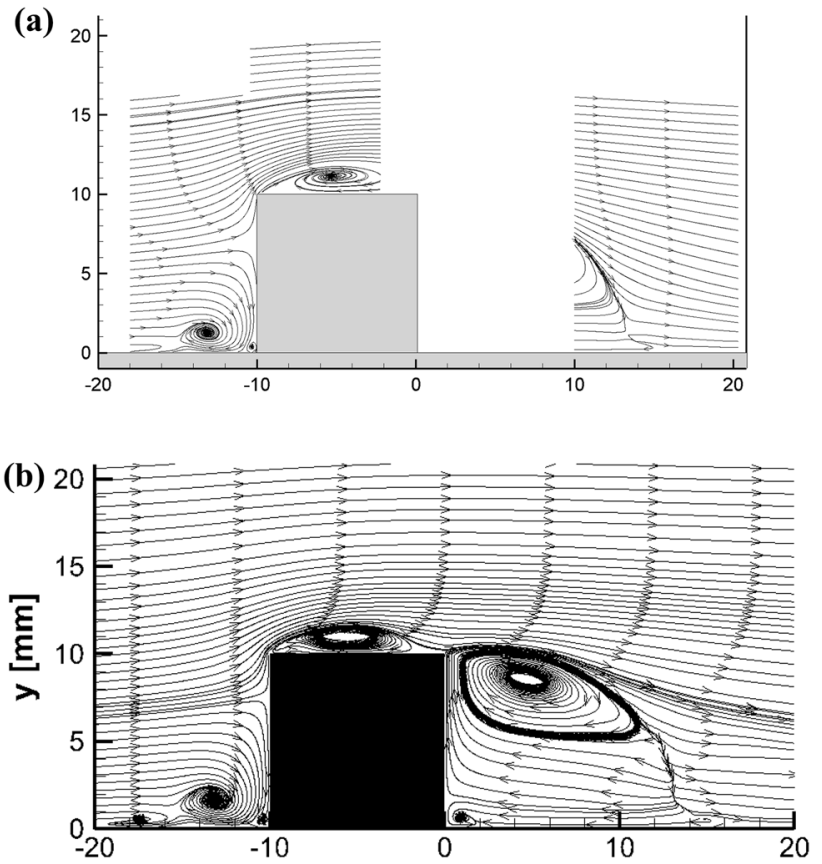

Fig. 25 Comparison between TR-PIV (a) and LBM results (b). The streamlines are plotted at the symmetry plane $z=0 \mathrm{~mm}$ for the turbulent test case at $U_{\infty}=0.8 \mathrm{~m} / \mathrm{s}$

spatial dynamic range, which is given with the positional accuracy of the individual tracks $(\approx 0.1$ pixel, here $\approx 4 \mu \mathrm{m})$ and limited only by the possible number of statistical independent samples per bin. By applying symmetry conditions even higher spatial resolutions can be achieved (Fig. 22).

In Fig. 23, left a wall-parallel slice of the bin averaging result of Fig. 21 is given at $y=0.5 \mathrm{~mm}$. For this specific case at $U_{\infty}=0.2 \mathrm{~m} / \mathrm{s}$ or $\operatorname{Re}_{H}=2000$ with $\delta / H=0.74$ the displayed flow structure at the wall is a good and direct approximation of the mean skin friction velocity vector distribution. The flow pattern and vector field of the near wall mean flow is very similar to the DNS results shown for $\mathrm{Re}_{H}=3000 \mathrm{in}$ the paper by (Diaz-Daniel et al. 2017, Fig. 1). For calculation of the overall flow induced drag the skin friction field could be integrated together with those at the surfaces of the cube itself. Access to a direct estimation of the surface
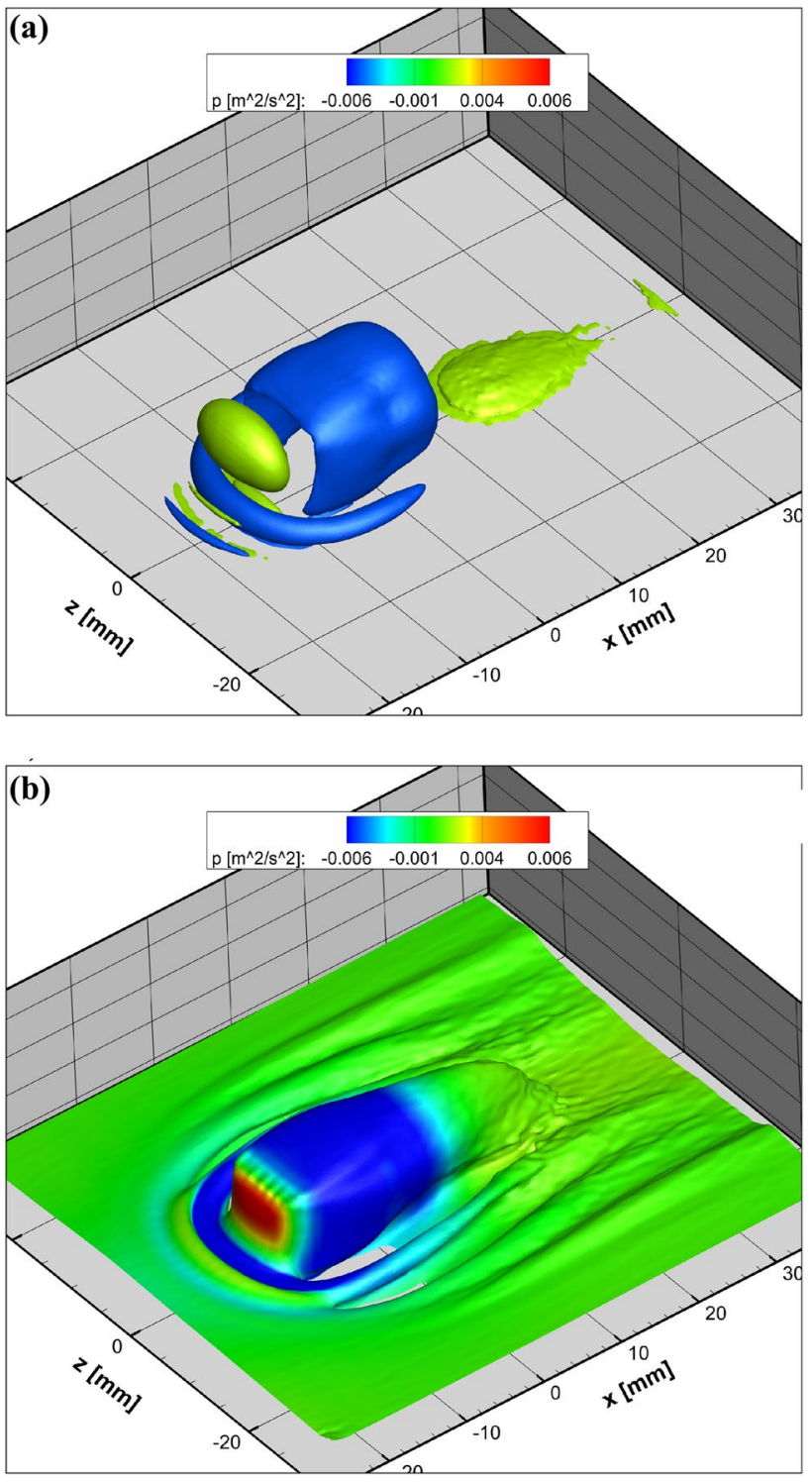

Fig. 26 Mean 3D pressure field iso-surfaces around the cube based on bin-averaging (a) and the same pressure field color coded on isosurfaces of mean streamwise velocity (b)

drag based on the flow field around an obstacle is considered a very interesting novelty for aerodynamic investigations. Together with the pressure field estimation, based on the same bin averaging result using the FlowFit approach in the entire volume (see Fig. 26), the classical drag estimation approach based on a wake integration of the pressure deficit can be tested and validated at different downstream wake distances allowing a quantitative comparison to the direct drag integration proposed here. This proposed approach might be addressed in a future campaign. It has not been attempted here because the volume size was not sufficient in span- and streamwise directions and the very small bin size (at least in wall-normal direction) required for estimating 
the average skin friction velocity field at all model surfaces would require a significantly higher number of samples to achieve statistical convergence.

A direct comparison of the streamlines upstream of the cube obtained from the TR-PIV measurement 3D bin-averaging and 3D STB data is provided in Fig. 24 for $U_{\infty}=0.2 \mathrm{~m} / \mathrm{s}$ in the center-plane. Furthermore a comparison between the time-averaged TR-PIV measurements and the LBM simulation is provided in Fig. 25, for the turbulent case at $U_{\infty}=0.8 \mathrm{~m} / \mathrm{s}$. The upstream horseshoe vortex, the separated flows at the corners and the recirculation region sizes are well defined and reproduced by the simulation. The deceleration of the flow below the cube's stagnation point generates a hierarchy of horse-shoe vortices with negative spanwise vorticity close to the wall. In between the rollers smaller counter-rotating vortices are induced with reduced vorticity magnitudes. In the $3 \mathrm{D}$ bin-averaged result the streamlines of the first-order and strongest horse-shoe vortex closest to the cubes front surface "escape" in spanwise direction (empty core) due to a small asymmetry of the averaged flow. This might be caused by the low frequency quasiperiodic oscillations in the wake downstream of the cube or by the inherent asymmetries of bluff-body wakes (Fig. 26).

\subsection{Reynolds number effects for the laminar test cases}

With increasing Reynolds numbers the flow around the cube changes mainly for three reasons: (a) the momentum of the incoming flow increases, while (b) at the same time the laminar boundary layer thickness decreases and (c) the dynamics of the transitional and turbulent flow structures along the shear layers and in the wake increases. At higher flow velocities this leads to higher accelerations at the cube front edges and consequently to an increase of the flow separation domain size around the cube's side and top faces as well as in the wake. The volume of negative mean streamwise velocities $u$, indicated by the blue iso-contour surfaces in Fig. 22, increase both in spanwise-as well as in streamwise direction with increasing Reynolds number. Furthermore, the extracted horizontal plane close to the wall shows an increasing upstream extension of an area of negative streamwise velocities for the higher Reynolds number cases indicating the influence of the first, second and third generation of horse-shoe vortices induced in front of the cube (see Fig. 24). In the same extracted horizontal plane and in the middle crossflow plane ( $Y Z$-plane) the increase of the wall-normal momentum exchange due to the presence of the streamwise aligned lateral horseshoe vortices can be clearly seen. More general flow features can also be observed in the 3D flow bin-averaged images of Fig. 22.

The transitional and turbulent flow structures in particular (e.g., wavy and hairpin-like vortices in Figs. 17 and 18) which are developing along the shear layers enveloping the negative $u$-velocity values and along the lateral cube faces the horse-shoe vortices change their character in the wake of the cube with increasing Reynolds number. As the range of scales and the amplitude of Reynolds stress events, which guide the momentum exchange through the mean shear layers, increase with Reynolds number, also the dynamics of the flow separation region in the wake is affected: for the two steps from $\mathrm{Re}_{H}=2000$ to 4000 and 6000 , a significant streamwise increase of the separation bubble in the cube wake can be observed with mean reattachment point moving from $x / H=1.2$ to 1.8 ). Increasing the conditions from $\operatorname{Re}_{H}=6000-8000$ only a small shift of the reattachment point is observed by moving from $x / H=1.8$ to 1.85 ). This can be explained for the first two steps by the overall increase of the momentum deficit induced by the presence of the cube and the reduced turbulent exchange in the shear layers, while the last reduced step size is caused by a significant increase of momentum exchange driven by vortical structures (see Fig. 13) through the shear layers due to an already fully developed turbulent wake at the higher Reynolds numbers. A detailed view into the dynamics of the interaction of shear layer development and the complete wake would need further analysis of the different spectra for velocity and pressure fluctuations, preferably in 3D space. This would require a full set of time series of FlowFit data assimilation results together with the TR-PIV results combined with advanced modal decomposition analysis which are beyond the scope of the present paper and will be reserved for future work.

\subsection{TR-PIV versus 3D STB: Pros and Cons}

In the present experimental investigation two particle based measuring techniques were applied in a complementary manner regarding spatial (and temporal) resolution and statistical convergence of the measured quantities. TR-PIV measurements were performed locally to obtain velocity 'profiles' of the mean velocity fields and related higherorder statistics. Additionally, the spatio-temporally gridded data of the time-resolved $2 \mathrm{C}$ vector fields enable the determination of related frequency spectra. TR-PIV profile measurements can be done with a single camera setup at high magnifications with a simple 2D calibration and high sample rates. The combined advantages on the hardware side are an increase of the camera frame rate with the reduction of the CMOS camera sensor resolution in one dimension to a " $2 \mathrm{D}$ column" imaging particles within a narrow collimated the laser light sheet. The collimation allows the local light energy density to be increased sufficiently for the illumination of small tracer particles even at high repetition rates. The employed cross-correlation based PIV analysis is fast, robust and well established, but induces low-pass spatial filtering effects (Raffel et al. 2018, Ch.6). In future 
the spatial filtering can be reduced by applying a variant of the STB evaluation scheme specifically catered to handle 2D-2C particle image sequence data (Schröder et al. 2018). Here the spatial resolution for the mean and fluctuation statistics can be significantly increased by using advanced subpixel binning approaches (Godbersen and Schröder 2020). Furthermore, a 2D B-spline fit applied to the particle tracks similar to the FlowFit scheme could be used (even without physical regularization) in order to retrieve gridded data for computing the spectral content.

On the other hand the full volumetric Lagrangian particle tracking approach STB provides access to 3D topologies of the flow features together with Lagrangian particle transport mechanisms and their material derivatives around the cube in a randomly distributed manner. Position, velocity and acceleration of the scattered Lagrangian particle tracks are then used as input values for the data assimilation method FlowFit. The latter delivers time-series of 3D velocity (gradient) vector- and pressure fields which directly provide further quantities such as 3D vorticity or shear-strain. The spatial resolution for the instantaneous 3D STB and FlowFit results are depended on the maximum allowable mean particle distance and thus on the camera resolution and maximal possible solvable ppp-value for the STB tracking code. In the present case the rather macroscopic measurement volume was not able to sample the complete wave-number spectrum of the investigated transitional and turbulent flow field and wake structures. The same above mentioned advanced sub-pixel binning procedure can nonetheless provide highly resolved 3D mean and fluctuation statistics with a large dynamic spatial range (DSR), when a sufficient amount of statistical independent tracking data is available for achieving convergence in small 3D bins. Because the reconstructed track position accuracy of such experimental STB approaches are typically in the range of 0.1-0.15 pixel, the allowable bin sizes for such statistics are only limited by the position accuracy and the number of entries per bin required for reaching statistical convergence of various flow field quantities. The disadvantage of 3D STB is the complexity of the multiple camera arrangement, typically involving 4-8 cameras, and the high-power and high-repetition laser required for sufficient illumination of the imaged volume. Furthermore, the data quality strongly depends on careful 3D calibration procedures involving volume self calibration (VSC, Wieneke 2008) and calibration of the optical transfer function (OTF, Schanz et al. 2016). Ultimately, the volume size is limited by the available laser energy density per pulse (even with a mirrored multi-pass illumination strategy (Schröder et al. 2008; Ghaemi and Scarano 2010) along with the allowable size of particles to resolve all flow features without biases or truncation errors.

Both time-series of instantaneous velocity (and pressure) results can be used for subsequent modal analysis, such as proper orthogonal decomposition (POD) or dynamic mode decomposition (DMD), or for the detection and tracking of coherent flow structures. Specifically, analysis methods used for the study of lagrangian coherent structures could be applied to the 3D STB and FlowFit data due to the combined availability of Eulerian and Lagrangian flow field properties.

\section{Conclusions}

In the present paper we report on spatially and temporally highly resolved 2D and 3D velocity, pressure and acceleration field measurements of the flow around a surface mounted cube based on TR-PIV and 3D STB, the latter complemented with successive FlowFit data assimilation. From 3D STB as well dense Lagrangian particle tracks are available over long time sequences. Instantaneous flow features and dynamics in representative regions around the cube as well as converged flow statistics have been addressed for several Reynolds numbers at well characterized four laminar and one turbulent BL flow conditions. Mean flow statistics, volumetric flow structure organization and spectral data have been compared with experimental and numerical results given in the literature. Additionally, flow simulation using LBM have been performed for the turbulent test case and, with respect to the complex flow behavior, a relatively high degree of agreement with the experimental data can be stated.

As a result of this comprehensive flow investigation, an experimental data base is now available for further flow characterization and analysis tools. The data base consists of time-resolved and mean 2D and 3D velocity vector fields and volumes, corresponding 3D pressure fields and all six Reynolds stresses for all five test cases-incoming laminar $\mathrm{BL}$ at $U_{\infty}=0.2,0.4,0.6$ and $0.8 \mathrm{~m} / \mathrm{s}\left(\operatorname{Re}_{H}=2000-8000\right)$ and incoming turbulent $\mathrm{BL}$ at $U_{\infty}=0.8 \mathrm{~m} / \mathrm{s}$. Furthermore, time-resolved examples of STB results using FlowFit data assimilation and Lagrangian particle trajectories can be provided by DLR upon request for the validation advanced, time-resolved CFD methods (e.g., LES, DNS) using timeresolved 3D flow field data obtained from experiments.

Acknowledgements Open Access funding provided by Projekt DEAL. The authors would like to acknowledge the contribution of the experimental team at ONERA Lille to setup and run the THBV facility, assist in the associated measurements and provided useful support during the experimental campaign, namely Jacques Pruvost, Anne Gilliot and Jean-Claude Monnier.

Availability of data and material Time-averaged data are available by request from the first author. 


\section{Compliance with ethical standards}

Funding The presented work has been supported by the DLR-ONERA Common Research Project CUBE and partly funded by the Deutsche Forschungsgemeinschaft (DFG) through Grant No. SCHR 1165/5-1 as part of the Priority Programme on Turbulent Superstructures (DFG SPP 1881).

Conflict of Interest The authors declare that they have no conflict of interest.

Code availability PIV processing was performed with the PIVview software package (PIVTEC GmbH) whereas the STB analysis is based on a DLR in-house code. LBM simulations utilized a package developed by ONERA using the commercial ProLB solver.

Open Access This article is licensed under a Creative Commons Attribution 4.0 International License, which permits use, sharing, adaptation, distribution and reproduction in any medium or format, as long as you give appropriate credit to the original author(s) and the source, provide a link to the Creative Commons licence, and indicate if changes were made. The images or other third party material in this article are included in the article's Creative Commons licence, unless indicated otherwise in a credit line to the material. If material is not included in the article's Creative Commons licence and your intended use is not permitted by statutory regulation or exceeds the permitted use, you will need to obtain permission directly from the copyright holder. To view a copy of this licence, visit http://creativecommons.org/licenses/by/4.0/.

\section{References}

Beresh SJ, Henfling JF, Spillers RW, Spitzer SM (2018) 'Postagestamp PIV': small velocity fields at $400 \mathrm{kHz}$ for turbulence spectra measurements. Meas Sci Technol 29(3):034011. https://doi. org/10.1088/1361-6501/aa9f79

Castro IP, Robins AG (1977) The flow around a surface-mounted cube in uniform and turbulent streams. J Fluid Mech 79(2):307-335. https://doi.org/10.1017/S0022112077000172

Chen S, Doolen GD (1998) Lattice Boltzmann method for fluid flows. Annu Rev Fluid Mech 30(1):329-364. https://doi.org/10.1146/ annurev.fluid.30.1.329

Christensen KT (2004) The influence of peak-locking errors on turbulence statistics computed from PIV ensembles. Exp Fluids 36(3):484-497. https://doi.org/10.1007/s00348-003-0754-2

Cuvier C, Srinath S, Stanislas M, Foucaut JM, Laval JP, Kähler CJ, Hain R, Scharnowski S, Schröder A, Geisler R, Agocs J, Röse A, Willert C, Klinner J, Amili O, Atkinson C, Soria J (2017) Extensive characterisation of a high Reynolds number decelerating boundary layer using advanced optical metrology. J Turbul 18(10):929-972. https://doi.org/10.1080/14685248.2017.1342827

Depardon S, Lasserre JJ, Boueilh JC, Brizzi LE, Borée J (2005) Skin friction pattern analysis using near-wall PIV. Exp Fluids 39(5):805-818. https://doi.org/10.1007/s00348-005-0014-8

Depardon S, Lasserre JJ, Brizzi LE, Borée J (2006) Instantaneous skinfriction pattern analysis using automated critical point detection on near-wall PIV data. Meas Sci Technol 17(7):1659-1669. https ://doi.org/10.1088/0957-0233/17/7/004

Depardon S, Lasserre JJ, Brizzi LE, Borée J (2007) Automated topology classification method for instantaneous velocity fields. Exp Fluids 42(5):697-710. https://doi.org/10.1007/s00348-007-0277-3

Diaz-Daniel C, Laizet S, Vassilicos J (2017) Direct numerical simulations of a wall-attached cube immersed in laminar and turbulent boundary layers. Int J Heat Fluid Flow 68:269-280. https://doi. org/10.1016/j.ijheatfluidflow.2017.09.015

Gesemann S, Huhn F, Schanz D, Schröder A (2016) From noisy particle tracks to velocity, acceleration and pressure fields using B-splines and penalties. In: 18th International Symposium on Applications of Laser and Imaging Techniques to Fluid Mechanics, Lisbon, Portugal, http://ltces.dem.ist.utl.pt/lxlaser/lxlaser201 6/finalworks2016/papers/04.5_4_186paper.pdf

Ghaemi S, Scarano F (2010) Multi-pass light amplification for tomographic particle image velocimetry applications. Meas Sci Technol 21(12):127002. https://doi.org/10.1088/0957-0233/21/12/127002

Godbersen P, Schröder A (2020) Functional binning: improving convergence of Eulerian statistics from Lagrangian particle tracking. Meas Sci Technol. https://doi.org/10.1088/1361-6501/ab8b84

Huhn F, Schanz D, Manovski P, Gesemann S, Schröder A (2018) Timeresolved large-scale volumetric pressure fields of an impinging jet from dense Lagrangian particle tracking. Exp Fluids 59:81. https ://doi.org/10.1007/s00348-018-2533-0

Hussein HJ, Martinuzzi RJ (1996) Energy balance for turbulent flow around a surface mounted cube placed in a channel. Phys Fluids 8(3):764-780. https://doi.org/10.1063/1.868860

Iaccarino G, Ooi A, Durbin P, Behnia M (2003) Reynolds averaged simulation of unsteady separated flow. Int J Heat Fluid Flow 24(2):147-156. https://doi.org/10.1016/S0142-727X(02)00210-2

Islam S, Zhou C, Shah A, Xie P (2012) Numerical simulation of flow past rectangular cylinders with different aspect ratios using the incompressible lattice Boltzmann method. J Mech Sci Technol 26:1027-1041. https://doi.org/10.1007/s12206-012-0328-4

Krajnović S, Davidson L (2002) Large-eddy simulation of the flow around a bluff body. AIAA J 40(5):927-936. https://doi. org/10.2514/2.1729

Lim HC, Castro IP, Hoxey RP (2007) Bluff bodies in deep turbulent boundary layers: Reynolds-number issues. J Fluid Mech 571:97118. https://doi.org/10.1017/S0022112006003223

Lim HC, Thomas T, Castro IP (2009) Flow around a cube in a turbulent boundary layer: LES and experiment. J Wind Eng Ind Aerodyn 97(2):96-109. https://doi.org/10.1016/j.jweia.2009.01.001

Liu T, Liu G, Ge Y, Wu H, Wu W (2008) Extended lattice Boltzmann equation for simulation of flows around bluff bodies in high Reynolds number. In: 6th International Colloquium on Bluff Bodies Aerodynamics and Applications (BBAA VI), Milano, Italy

Lynch K, Scarano F (2013) A high-order time-accurate interrogation method for time-resolved PIV. Meas Sci Technol 24(3):035305. https://doi.org/10.1088/0957-0233/24/3/035305

Martinuzzi R, Tropea C (1993) The flow around surface-mounted, prismatic obstacles placed in a fully developed channel flow (data bank contribution). J Fluids Eng 115(1):85-92. https://doi. org/10.1115/1.2910118

Meinders ER, Hanjalic K, Martinuzzi RJ (1999) Experimental study of the local convection heat transfer from a wall-mounted cube in turbulent channel Flow. J Heat Transfer 121(3):564-573. https:// doi.org/10.1115/1.2826017

Novara M, Schanz D, Reuther N, Kähler CJ, Schröder A (2016) Lagrangian 3D particle tracking in high-speed flows: Shake-TheBox for multi-pulse systems. Exp Fluids 57(8):128. https://doi. org/10.1007/s00348-016-2216-7

Novara M, Schanz D, Geisler R, Gesemann S, Voss C, Schröder A (2019) Multi-exposed recordings for 3D Lagrangian particle tracking with multi-pulse Shake-The-Box. Exp Fluids 60(3):44. https://doi.org/10.1007/s00348-019-2692-7

Qian YH, D'Humières D, Lallemand P (1992) Lattice BGK models for Navier-Stokes equation. Europhys Lett 17(6):479-484. https ://doi.org/10.1209/0295-5075/17/6/001

Raffel M, Willert C, Kähler C, Scarano F, Wereley S, Kompenhans J (2018) Particle image velocimetry: a practical guide, 3rd edn. Springer, Berlin. https://doi.org/10.1007/978-3-319-68852-7 
Schanz D, Gesemann S, Schröder A, Wieneke B, Novara M (2012) Non-uniform optical transfer functions in particle imaging: calibration and application to tomographic reconstruction. Meas Sci Technol 24(2):024009. https://doi.org/10.1088/09570233/24/2/024009

Schanz D, Gesemann S, Schröder A (2016) Shake-The-Box: Lagrangian particle tracking at high particle image densities. Exp Fluids 57(5):70. https://doi.org/10.1007/s00348-016-2157-1

Schlatter P, Örlü R, Li Q, Brethouwer G, Fransson JHM, Johansson AV, Alfredsson PH, Henningson DS (2009) Turbulent boundary layers up to $R e_{\theta}=2500$ studied through simulation and experiment. Phys Fluids 21(5):051702. https://doi.org/10.1063/1.3139294

Schröder A, Geisler R, Elsinga G, Scarano F, Dierksheide U (2008) Investigation of a turbulent spot and a tripped turbulent boundary layer flow using time-resolved tomographic PIV. Exp Fluids 44:305-316. https://doi.org/10.1007/s00348-007-0403-2

Schröder A, Schanz D, Novara M, Philipp F, Geisler R, Agocs J, Knopp T, Schroll M, Willert CE (2018) Investigation of a high Reynolds number turbulent boundary layer flow with adverse pressure gradients using PIV and 2D- and 3D- Shake-The-Box. In: 19th International Symposium on Application of Laser and Imaging Techniques to Fluid Mechanics, Lisbon, Portugal, https://elib.dlr. de/121221/2/257_paper_LXLASER2018_FINAL.pdf

Tannoury E, Ricot D, Machrouki H, Sengissen A (2014) Validation of a new CFD solver based on the lattice Boltzmann method. In: 23rd International Conference on Discrete Simulation of Fluid Dynamics (DSFD), Paris, France

Touil H, Ricot D, Lévêque E (2014) Direct and large-eddy simulation of turbulent flows on composite multi-resolution grids by the lattice Boltzmann method. J Comput Phys 256:220-233. https:// doi.org/10.1016/j.jcp.2013.07.037

Wieneke B (2008) Volume self-calibration for 3D particle image velocimetry. Exp Fluids 45(4):549-556. https://doi.org/10.1007/ s00348-008-0521-5

Willert C, Cuvier C, Foucaut J, Klinner J, Stanislas M, Laval J, Srinath S, Soria J, Amili O, Atkinson C, Kähler C, Scharnowski S, Hain R, Schröder A, Geisler R, Agocs J, Röse A (2018) Experimental evidence of near-wall reverse flow events in a zero pressure gradient turbulent boundary layer. Exp Therm Fluid Sci 91:320-328. https://doi.org/10.1016/j.expthermflusci.2017.10.033

Willert CE (2015) High-speed particle image velocimetry for the efficient measurement of turbulence statistics. Exp Fluids 56(1):17. https://doi.org/10.1007/s00348-014-1892-4

Yakhot A, Anor T, Liu H, Nikitin N (2006a) Direct numerical simulation of turbulent flow around a wall-mounted cube: spatio-temporal evolution of large-scale vortices. J Fluid Mech 566:1-9. https ://doi.org/10.1017/S0022112006002151

Yakhot A, Liu H, Nikitin N (2006b) Turbulent flow around a wallmounted cube: a direct numerical simulation. Int J Heat Fluid Flow 27(6):994-1009. https://doi.org/10.1016/j.ijheatfluidflow .2006 .02 .026

Publisher's Note Springer Nature remains neutral with regard to jurisdictional claims in published maps and institutional affiliations. 